\title{
Article \\ Local and Global Stability Analysis of Dengue Disease with Vaccination and Optimal Control
}

\author{
Anusit Chamnan ${ }^{1}$, Puntani Pongsumpun ${ }^{1, * \mathbb{C}}$, I-Ming Tang ${ }^{2}$ and Napasool Wongvanich ${ }^{3}$ \\ 1 Department of Mathematics, School of Science, King Mongkut's Institute of Technology Ladkrabang, \\ Bangkok 10520, Thailand; 62605005@kmitl.ac.th \\ 2 Department of Physics, Faculty of Science, Mahidol University, Bangkok 10400, Thailand; \\ imingtang@yahoo.com \\ 3 Department of Instrumentation and Control Engineering, School of Engineering, King Mongkut's Institute of \\ Technology Ladkrabang, Bangkok 10520, Thailand; napasool.wo@kmitl.ac.th \\ * Correspondence: puntani.po@kmitl.ac.th; Tel.: +66-2329-8000
}

Citation: Chamnan, A.;

Pongsumpun, P.; Tang, I.-M.;

Wongvanich, N. Local and Global Stability Analysis of Dengue Disease with Vaccination and Optimal Control. Symmetry 2021, 13, 1917. https://doi.org/10.3390/sym13101917

Academic Editors: Antonio Palacios and Chiara Bedon

Received: 4 September 2021

Accepted: 8 October 2021

Published: 12 October 2021

Publisher's Note: MDPI stays neutral with regard to jurisdictional claims in published maps and institutional affiliations.

Copyright: (C) 2021 by the authors. Licensee MDPI, Basel, Switzerland. This article is an open access article distributed under the terms and conditions of the Creative Commons Attribution (CC BY) license (https:/ / creativecommons.org/licenses/by/ $4.0 /)$.

\begin{abstract}
Dengue fever is a disease that has spread all over the world, including Thailand. Dengue is caused by a virus and there are four distinct serotypes of the virus that cause dengue DENV-1, DENV-2, DENV-3, and DENV-4. The dengue viruses are transmitted by two species of the Aedes mosquitoes, the Aedes aegypti, and the Aedes albopictus. Currently, the dengue vaccine used in Thailand is chimeric yellow tetravalent dengue (CYD-TDV). This research presents optimal control which studies the vaccination only in individuals with a documented past dengue infection (seropositive), regardless of the serotypes of infection causing the initial infection by the disease. The analysis of dengue transmission model is used to establish the local asymptotically stabilities. The property of symmetry in the Lyapunov function an import role in achieving this global asymptotically stabilities. The optimal control systems are shown in numerical solutions and conclusions. The result shows that the control resulted in a significant reduction in the number of infected humans and infected vectors.
\end{abstract}

Keywords: dengue fever; global asymptotically stabilities; local asymptotically stabilities; optimal control; vaccination

\section{Introduction}

Mosquito-borne dengue fever viral disease infects hundreds of millions of people in tropical and subtropical areas every year [1]. Thailand has reported outbreaks of dengue fever for more than 60 years. At present, dengue fever has spread throughout the country, every province, and every district [2]. Dengue hemorrhagic fever is contagious with the Aedes aegypti mosquito being an important disease-carrying insect in many rural areas. The Aedes albopictus is a second disease-carrying mosquito. The four virus serotypes that cause dengue virus (DENV) are DENV-1, DENV-2, DENV-3, and DENV-4 [3-5]. In Thailand, the Aedes aegypti mosquito is considered to be the primary vector of the dengue virus. Infection with dengue serotype provides lifelong immunity to that serotype and will have cross-protection against other species heterotypic immunity for a short period of time [6,7]. The clinical symptoms of dengue virus infection range from being asymptomatic to severe illness that can lead to death if not properly treated. In most cases involving young children, there is a mild fever with a red rash and the child is said to be suffering from blood fever. In older children teenagers and adults, the symptoms may be worse, i.e., they may come down with high fevers, thrombocytopenia, leukopenia, rashes, myalgia, and arthralgia. Dengue fever (DF) is more common in children under 15 years of age. In areas in which hyperemia is also occurring, there may be recurrent dengue infections during which the more severe symptoms are occurring, and the patients may be suffering from dengue hemorrhagic fever (DHF). If the plasma leakage becomes extremely severe, the illness develops into dengue shock syndrome (DSS) [8-10] and the patient might die. 
The incidence of dengue fever infection appears to be age dependent. Pongsumpun and Tang [11] reported that in one province in Thailand in 1998, mentioned that most of the cases occurred in children below the age of 15. Sriprom et al. [12] mentioned that this did not change much. What changed over time was the dengue strain responsible for the infection, very few of the dengue infections were due to DENV-4. In the last few years, the greatest number of infections is caused by the DENV-4 strain. We have not taken this change into account. Most infections caused by the dengue virus and other viruses lead are either asymptomatic or minimally symptomatic with no way to tell whether the patients were infected or not. Burke et al. [13] reported that $87 \%$ of the tested infected children in Bangkok Thailand in 1980-1981 belong to these two groups. We do not expect much had changed over the years except for the strain of the dengue virus.

A vaccine to prevent dengue fever has recently been developed. The vaccine efficacy of the dengue vaccine was moderate at $56.5 \%$, meaning it could prevent more than half the number of dengue infections. The dengue vaccine reductions in hospital admissions by $80.3 \%$ [14], and 67.2\% [15]. The CYD-TDV vaccine is highly effective among children with serological evidence of prior DENV infection. A Phase 3 study of dengue vaccine found that dengue vaccine was able to reduce the severity of the disease by $88.5 \%$ and prevent dengue by $90.0 \%$ [16]. A cautionary note should be mentioned, there is an increased risk of hospitalization of the vaccinated individual among groups without prior infections. Mass vaccination with the CYD-TDV vaccine should not be undertaken in countries that do not have a history of dengue infections by most of the strains of the DENV. This is why the vaccination programs involving this vaccine have been stopped in countries such as Thailand, where there is a prior history of infections by multiple strains of the DENV's.

The chimeric yellow fever dengue virus tetravalent dengue vaccine (CYD-TDV) covers four serotypes of dengue. The Dengue vaccination program requires a total of three injections, 6 months apart at 0, 6, and 12 months. The optimal age for injection is over 9 years [17]. A single dose of dengue vaccination is as effective as the three-dose vaccination program. This may be due to the fact that $79 \%$ of the cohort in the study had dengue seropositivity at baseline in countries with dengue-endemic areas [18]. The immunization obtained from vaccination gradually loses as time goes on. Once infected with any serotypes, the body will always be immune to those serotypes. However, they are temporarily immune to other serotypes, thus there is a chance of re-infecting dengue in other serotypes until all are complete.

The World Health Organization (WHO) aims to reduce the mortality and morbidity of dengue by at least 50\% and 25\%, respectively, by 2020 [19]. Esteva and Vargus [20] studied local stability and global stability of models with the susceptible-infected-recovery (SIR) model of dengue transmission and assumed that the human population and the vector population are constant. Chanprasopchai et al. [21] studied dengue fever and the effect of vaccination with analyzed of stable conditions of an equilibrium point of the model using Routh-Hurwitz criteria. A mathematical model of dengue with and without awareness in the host population [22] assumes that some hosts do not interact with infected mosquitoes. This is because they take different preventive measures due to their perception of the disease. $\mathrm{Wu}$ and Wong [23] studied the mathematical model with two delays to reflect the extrinsic and intrinsic incubation periods of virus in the dengue disease transmission model. The stability analysis of two equilibrium points is carried out and a simulation is given for different parameter settings and to simulate the succession of two epidemics with variable human populations [24]. Khan and Fatmawati [25] described changes in dengue infection from basic reproductive values which defines a model with hospitalization and presents the changes in detail. The results suggest that spraying insecticides on mosquitoes can significantly reduce dengue infection. Pongsumpun et al. [26] studied a mathematical model of the dengue model with a vertical transmission control mechanism based on the dengue model. The two policy control models were insecticide and vaccinations which simulated the parameters affecting this model control. The authors [27] study a dynamic basic mathematical model for dengue transmission in Thailand, by including infection of 
different serotypes to analyze the effects of different vaccination strategies. With an interest in the optimal control between primary and secondary infection. There are many studies about applying to solve the optimal control problem of dengue fever [28-32].

In Thailand, data on dengue hemorrhagic fever (DHF) comes from the Department of Disease Control, the Ministry of Health. It is seen that dengue hemorrhagic fever is prevalent in 2013 per 100,000 which is the highest and the number of morbidities will trend fluctuated each year from 2003 to 2020. Likewise, when the number of mortality direct variation with the number of morbidities, but this is a very small percentage compared to the infected as shown in Figure 1.

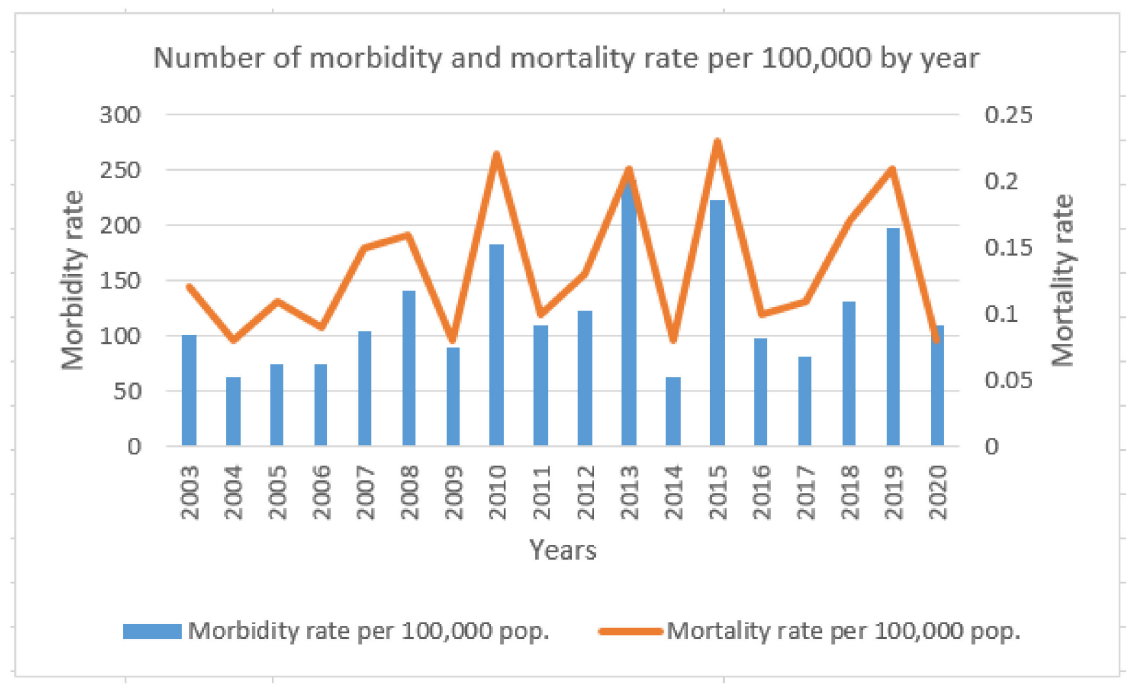

Figure 1. The number of morbidity and mortality rate per 100,000 by year from 2003-2020 [33].

In this paper, we study the vaccination only in individuals with a documented past dengue infection (seropositive), regardless of the serotypes of infection, with a simplified model. This model focused on the population of Thailand for the provinces with high morbidity rates, such as Mae Hong Son, Rayong, Nakhon Ratchasima, Chaiyaphum, Chainat, and the regions with the highest morbidity rates were the Northeast, North, Central, Southern regions, respectively, where there were outbreaks of dengue hemorrhagic fever, resulting in people who have been infected with dengue fever being re-infected. The human population is modeled through the SEIRS framework, and the vector population is modeled using the SI model. Local and global stability analyses are given with the basic reproduction number being the bifurcation point. The property of symmetry in the Lyapunov function an important role in achieving this global asymptotically stabilities. The optimal control is applied in the spreading model to minimizes the number of infected human and infected vector populations. The results are then given numerically to demonstrate the analyses.

\section{Materials and Methods}

The mathematical model of the dengue transmission is for the vector population and the human population. The susceptible-infected (SI) and susceptible-exposed-infectedrecovered (SEIRS) model for vector and human population, respectively. In Thailand, some regions are densely populated with a high prevalence of Aedes mosquitoes. This model of dengue outbreak considers vaccination only in previously infected populations as it reduces the severity of the disease and reduces the rate of hospitalization for the next infection. Note that dengue fever has four different serotypes, and we can get infected up to four times. Vaccination should be administered after each injection to minimize the risk of hospitalization and the severity of the disease in following infections. Once infected with any dengue serotype, the person is immune to that serotype for life and only has a short period of time of cross-protection against other species, as advised by the World 
Health Organization. In this model, the transmission of the dengue virus when vaccinated after each infection is described. The human and vector populations are divided into four and two individuals, respectively. The variables and parameters are defined listed in Table 1 . The dynamic dengue fever model with vaccination after each infectious is shown in Figure 2.

Table 1. The parameters used in the numerical simulation.

\begin{tabular}{|c|c|}
\hline Variables and Parameters & Biological Meaning \\
\hline$\overline{S_{H}}$ & The number of susceptible human population \\
\hline$\overline{S_{V}}$ & The number of susceptible vector population \\
\hline$\frac{\overrightarrow{E_{H}}}{\mathrm{E}}$ & The number of exposed human population \\
\hline$\overline{I_{H}}$ & The number of infected human population \\
\hline$\frac{I_{V}}{I_{V}}$ & The number of infected vector population \\
\hline$\frac{v}{R_{H}}$ & The number of recovered vector population \\
\hline$P$ & The vaccine efficiency \\
\hline$\beta_{H}$ & The transmission rate of dengue virus from vector to the human \\
\hline$\beta_{V}$ & The transmission rate of dengue virus from the human to vector \\
\hline$\alpha$ & The biting rate of vector population \\
\hline$\theta$ & The recurrent infection rate \\
\hline$\varepsilon$ & The incubation rate \\
\hline$\gamma$ & The recovery rate \\
\hline$A$ & The constant recruitment rate \\
\hline$d_{H}$ & The natural mortality rate of the human population \\
\hline$d_{d}$ & The mortality rate from infection of the human population \\
\hline$d_{V}$ & The natural mortality rate of the vector population \\
\hline$d_{k}$ & The mortality rate from infection of the vector population \\
\hline$b_{H}^{n}$ & The birth rate \\
\hline$N_{H}$ & The total number of the human population \\
\hline$N_{V}$ & The total number of the vector population \\
\hline
\end{tabular}
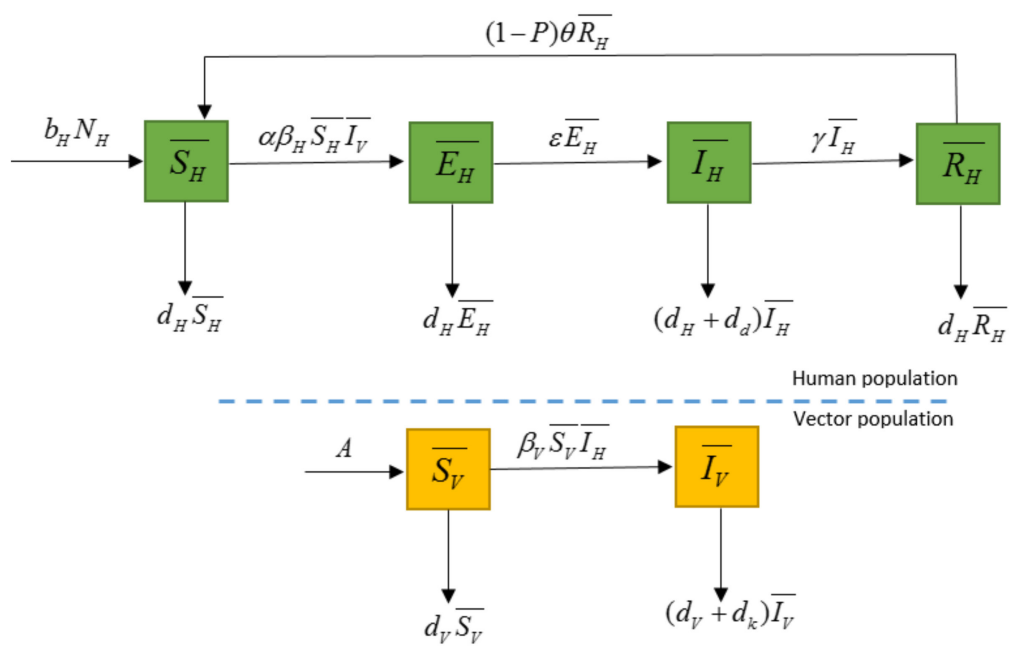

Figure 2. Schematic diagram representation compartments vector and human population after each infectious dengue fever.

The dynamics behavior model of human and vector population is encapsulated in the following systems of differential equations:

$$
\begin{gathered}
\frac{d \overline{S_{H}}}{d t}=b_{H} N_{H}+(1-P) \theta \overline{R_{H}}-\alpha \beta_{H} \overline{S_{H} I_{V}}-d_{H} \overline{S_{H}} \\
\frac{d \overline{E_{H}}}{d t}=\alpha \beta_{H} \overline{S_{H} \overline{I_{V}}}-\varepsilon \overline{E_{H}}-d_{H} \overline{E_{H}}
\end{gathered}
$$




$$
\begin{gathered}
\frac{d \overline{I_{H}}}{d t}=\varepsilon \overline{E_{H}}-\gamma \overline{I_{H}}-\left(d_{H}+d_{d}\right) \overline{I_{H}} \\
\frac{d \overline{R_{H}}}{d t}=\gamma \overline{I_{H}}-(1-P) \theta \overline{R_{H}}-d_{H} \overline{R_{H}} \\
\frac{d \overline{S_{V}}}{d t}=A-\beta_{V} \overline{S_{V} I_{H}}-d_{V} \overline{S_{V}} \\
\frac{d \overline{I_{V}}}{d t}=\beta_{V} \overline{S_{V} I_{H}}-\left(d_{V}+d_{k}\right) \overline{I_{V}}
\end{gathered}
$$

with the conditions

$$
\begin{gathered}
\overline{S_{H}}+\overline{E_{H}}+\overline{I_{H}}+\overline{R_{H}}=N_{H} \\
\overline{S_{V}}+\overline{I_{V}}=N_{V}
\end{gathered}
$$

The rate of change of the total population of human and vector are as follows:

$$
\begin{gathered}
\frac{d \overline{S_{H}}}{d t}+\frac{d \overline{E_{H}}}{d t}+\frac{d \overline{I_{H}}}{d t}+\frac{d \overline{R_{H}}}{d t}=0 \\
\frac{d \overline{S_{V}}}{d t}+\frac{d \overline{I_{V}}}{d t}=0
\end{gathered}
$$

The conditions of Equations (7) and (8) employ:

$$
\begin{gathered}
\overline{I_{H}}=\frac{\left(b_{H}-d_{H}\right) N_{H}}{d_{d}} \\
\overline{I_{V}}=\frac{A-d_{V} N_{V}}{d_{k}}
\end{gathered}
$$

Normalizing the equations with assuming the proportion number of the individual following as:

$$
\begin{gathered}
S_{H}=\frac{\overline{S_{H}}}{N_{H}}, E_{H}=\frac{\overline{E_{H}}}{N_{H}} I_{H}=\frac{\overline{I_{H}}}{N_{H}}, R_{H}=\frac{\overline{R_{H}}}{N_{H}} \\
S_{V}=\frac{\overline{S_{V}}}{N_{V}}, I_{V}=\frac{\overline{I_{V}}}{N_{V}}
\end{gathered}
$$

we also have

$$
\begin{gathered}
S_{H}+E_{H}+I_{H}+R_{H}=1 \\
S_{V}+I_{V}=1
\end{gathered}
$$

The normalized system of equations is:

$$
\left.\begin{array}{rl}
\frac{d S_{H}}{d t} & =b_{H}+(1-P)\left(1-S_{H}-E_{H}-I_{H}\right) \theta-\alpha \beta_{H} S_{H} I_{V} N_{V}-d_{H} S_{H} \\
\frac{d E_{H}}{d t} & =\alpha \beta_{H} S_{H} I_{V} N_{V}-\varepsilon E_{H}-d_{H} E_{H} \\
\frac{d I_{H}}{d t} & =\varepsilon E_{H}-\gamma I_{H}-\left(d_{H}+d_{d}\right) I_{H} \\
\frac{d I_{V}}{d t} & =\beta_{V}\left(1-I_{V}\right) I_{H} N_{H}-\left(d_{V}+d_{k}\right) I_{V}
\end{array}\right\}
$$

\section{Analysis of the Model}

\subsection{Positivity Invariant Sets of the Model}

Proposition 1. From [34], let $\left(S_{H}(t), E_{H}(t), I_{H}(t), R_{H}(t), S_{V}(t), I_{V}(t)\right)$ be the solution of Equations (1)-(6) with positive initial conditions $S_{H}(0), E_{H}(0), I_{H}(0), R_{H}(0), S_{V}(0), I_{V}(0)$. Denoting also the invariant set $\phi=\left\{\left(S_{H}, S_{V}, E_{H}, I_{H}, I_{V}, R_{H}\right) \in \mathbb{R}_{+}^{6}: N_{H} \leq \frac{b_{H}}{d_{H}}, N_{V} \leq \frac{A}{d_{V}}\right\}$ then the closed set $\phi$ is positive invariant that attracts all solutions in the space $\mathbb{R}_{+}^{6}$. 
Proof. We begin by setting $N_{T}(t)=\left(N_{H}(t), N_{V}(t)\right)=\left(S_{H}+E_{H}+I_{H}+R_{H}, S_{V}+I_{V}\right)$. The differentiation yields:

$$
\begin{gathered}
\frac{d N_{T}(t)}{d t}=\left(\frac{d N_{H}(t)}{d t}, \frac{d N_{V}(t)}{d t}\right) \\
=\left(b_{H}-d_{H} S_{H}-d_{H} E_{H}-d_{H} I_{H}-d_{d} I_{H}-d_{H} R_{H}, A-d_{V} S_{V}-d_{V} I_{V}-d_{k} I_{V}\right) \\
=\left(b_{H}-d_{H} N_{H}-d_{d} I_{H}, A-d_{V} N_{V}-d_{k} I_{V}\right) \\
\leq\left(b_{H}-d_{H} N_{H}, A-d_{V} N_{V}\right)
\end{gathered}
$$

Hence, $\frac{d N_{H}(t)}{d t}=b_{H}-d_{H} N_{H} \leq 0$ for $N_{H}(t) \geq \frac{b_{H}}{d_{H}}$ and $\frac{d N_{V}(t)}{d t}=A-d_{V} N_{V} \leq 0$ for $N_{V}(t) \geq \frac{A}{d_{V}}$. Then $\frac{d N_{T}(t)}{d t} \leq 0$ wherever $N_{H}(t) \geq \frac{b_{H}}{d_{H}}$ and $N_{V}(t) \geq \frac{A}{d_{V}}$. Integrating the above equation, it follows that $\frac{d N_{T}(t)}{d t} \leq 0$ on $0 \leq N_{H}(t), N_{V}(t) \leq\left(\frac{b_{H}}{d_{H}}+N_{H}(0) e^{-d_{H} t}\right.$, $\left.\frac{A}{d_{V}}+N_{V}(0) e^{-d_{V} t}\right)$. As $t \rightarrow \infty, e^{-d_{H} t} \rightarrow 0, e^{-d_{V} t} \rightarrow 0$, and then $0 \leq N_{H}(t), N_{V}(t) \leq\left(\frac{b_{H}}{d_{H}}, \frac{A}{d_{V}}\right)$. Since the epidemiological parameters are assumed to be positive, it follows that the region of all solutions of $\phi$ will be in $\mathbb{R}_{+}^{6}$. Thus $\phi$ is a positively invariant set. Note that all equations described by Equations (1)-(6) in the non-negative octant $\mathbb{R}_{+}^{6}$ are positively invariant [34-36].

\subsection{Equilibrium Points of the Model}

Let us find equilibrium points of system Equation (17) that describe the model. Note that by setting the right-hand side of system Equation (17) to zero yields two equilibrium points, namely:

The disease-free equilibrium point:

$$
E_{1}=(1,0,0,0)
$$

The endemic equilibrium point:

$$
\begin{aligned}
E_{2}= & \left(S_{H}^{*}, E_{H}^{*}, I_{H}^{*}, I_{V}^{*}\right) \text { with } \\
S_{H}^{*} & =\frac{\left(\pi_{1} \pi_{2}\left(\left(\gamma \varepsilon-\pi_{3} \theta+\gamma d_{H}+\left(\varepsilon+\pi_{4}\right) \pi_{5}\right) d_{K}-\left(\gamma \varepsilon-\pi_{3} \theta+\gamma d_{H}+\left(\varepsilon+\pi_{4}\right) \pi_{5}\right) d_{V}-\varepsilon \pi_{8} N_{H} \beta_{V}\right)\right)}{\varepsilon N_{H}\left(\left(\pi_{7}-d_{H}\right) \pi_{1} \pi_{2}-\alpha\left(\gamma \varepsilon-\pi_{3} \theta+\gamma d_{H}+\left(\varepsilon+\pi_{4}\right) \pi_{5}\right) N_{V} \beta_{H}\right) \beta_{V}} \\
E_{H}^{*} & =\frac{\pi_{2}\left(\pi_{1} \pi_{2} \pi_{4} \pi_{6}-\alpha \varepsilon\left(\pi_{7}-b_{H}\right) N_{H} N_{V} \beta_{H} \beta_{V}\right)}{\varepsilon N_{H}\left(\pi_{1} \pi_{2} \pi_{4}+\alpha\left(\gamma \varepsilon-\pi_{3} \theta+\gamma d_{H}+\left(\varepsilon+\pi_{4}\right) \pi_{5}\right) N_{V} \beta_{H}\right) \beta_{V}} \\
I_{H}^{*} & =\frac{\pi_{1} \pi_{2} \pi_{4} \pi_{6}-\alpha \varepsilon\left(\pi_{7}-b_{H}\right) N_{H} N_{V} \beta_{H} \beta_{V}}{N_{H}\left(\pi_{1} \pi_{2} \pi_{4}+\alpha\left(\gamma \varepsilon-\pi_{3} \theta+\gamma d_{H}+\left(\varepsilon+\pi_{4}\right) \pi_{5}\right) N_{V} \beta_{H}\right) \beta_{V}} \\
I_{V}^{*} & =\frac{\left(\pi_{7}-d_{H}\right) \pi_{1} \pi_{2} \pi_{6}+\alpha \varepsilon \pi_{8} N_{H} N_{V} \beta_{H} \beta_{V}}{\alpha N_{V} \beta_{H}\left(\left(\gamma \varepsilon-\pi_{3} \theta+\gamma d_{H}+\left(\varepsilon+\pi_{4}\right) \pi_{5}\right) \pi_{6}+\varepsilon \pi_{8} N_{H} \beta_{V}\right)}
\end{aligned}
$$

where

$$
\begin{gathered}
\pi_{1}=\left(\varepsilon+d_{H}\right), \pi_{2}=\left(\gamma+d_{H}+d_{d}\right), \pi_{3}=(P-1)(\gamma+\varepsilon), \pi_{4}=\left(\theta-P \theta+d_{H}\right), \\
\pi_{5}=\left(d_{H}+d_{d}\right), \pi_{6}=\left(d_{V}+d_{k}\right), \pi_{7}=(P-1) \theta, \pi_{8}=\left(\theta-P \theta+b_{H}\right) .
\end{gathered}
$$

\subsection{Basic Reproductive Number}

The next-generation matrix method of the works in $[37,38]$ is used to compute the basic reproductive number $\left(R_{0}\right)$ for the dengue model of system Equation (17). The classes $E_{H}, I_{H}$, and $I_{V}$ are identified as influencing the new infections. The $F$ and $V$ matrices are obtained as follows:

$$
F=\left[\begin{array}{ccc}
0 & 0 & \alpha \beta_{H} S_{H} N_{V} \\
0 & 0 & 0 \\
0 & \beta_{V} S_{V} N_{H} & 0
\end{array}\right], V=\left[\begin{array}{ccc}
d_{H}+\varepsilon & 0 & 0 \\
-\varepsilon & d_{H}+d_{d}+\gamma & 0 \\
0 & 0 & d_{V}+d_{k}
\end{array}\right]
$$




$$
V^{-1}=\left[\begin{array}{ccc}
\frac{1}{\left(d_{H}+\varepsilon\right)} & 0 & 0 \\
\frac{\varepsilon d_{V}+\varepsilon d_{k}}{\left(\varepsilon+d_{H}\right)\left(\gamma+d_{H}+d_{d}\right)\left(d_{V}+d_{k}\right)} & \frac{1}{\left(\gamma+d_{H}+d_{d}\right)} & 0 \\
0 & 0 & \frac{1}{\left(d_{V}+d_{k}\right)}
\end{array}\right]
$$

The basic reproductive number is obtained through the spectral radius of the matrix $R_{0}=\rho F V^{-1}$ is called the reproductive number and given by:

$$
R_{0}=\sqrt{\frac{\alpha \varepsilon\left((P-1) \theta-b_{H}\right) N_{H} N_{V} \beta_{H} \beta_{V}}{\left((P-1) \theta-d_{H}\right)\left(\gamma+d_{H}+d_{d}\right)\left(\varepsilon+d_{H}\right)\left(d_{V}+d_{k}\right)}}
$$

\subsection{Local Stability Analysis}

In this section, we are going to analyze the stability condition of two equilibrium points, namely the disease-free $E_{1}$ and the endemic $E_{2}$ equilibrium points. The outcome of stability condition analysis of these equilibria is shown in the following-Theorems 1 and 2. The obtained Jacobian matrix is:

$$
J_{E_{i}}=\left[\begin{array}{cccc}
(P-1) \theta-d_{H}-\alpha \beta_{H} I_{V} N_{V} & (P-1) \theta & (P-1) \theta & -\alpha \beta_{H} S_{H} N_{V} \\
\alpha \beta_{H} I_{V} N_{V} & -\varepsilon-d_{H} & 0 & 0 \\
0 & \varepsilon & -\gamma-d_{H}-d_{d} & 0 \\
0 & 0 & \beta_{V} N_{H}-\beta_{V} N_{H} I_{V} & -\beta_{V} N_{H} I_{H}-d_{V}-d_{k}
\end{array}\right]
$$

Theorem 1. If $R_{0}<1$, then the disease-free equilibrium point $E_{1}$ of Equations (17)-(20) is locally asymptotically stable and unstable otherwise.

Proof. The characteristic polynomial of the Jacobian matrix of Equation (20) evaluated at the disease-free equilibrium is:

$$
\operatorname{det}\left(J_{E_{1}}-\lambda I\right)=0
$$

The eigenvalues $(\lambda)$ are obtained from the roots of Equation (21) where $I$ is identity matrix $(4 \times 4)$ the matrix is:

$$
\begin{aligned}
& \lambda_{1}=-\left(\varepsilon+d_{H}\right), \\
& \lambda_{2}=-\left((P+1) \theta+d_{H}\right), \\
& \lambda_{3}=-\left(d_{H}+d_{d}+\gamma\right), \\
& \lambda_{4}=-\left(d_{V}+d_{k}\right) .
\end{aligned}
$$

It is obvious that all the eigenvalues have negative real part. Therefore, $E_{1}$ is locally asymptotically stable [38].

Theorem 2. The endemic equilibrium point $E_{2}$ is locally asymptotically stable if $R_{0}>1$ and unstable otherwise.

Proof. The characteristic equation of the Jacobian matrix evaluated at the endemic equilibrium is:

$$
\operatorname{det}\left(J_{E_{2}}-\lambda I\right)=0
$$

One eigenvalue of the above matrix is $\lambda=-\left(d_{H}+d_{d}+\gamma\right)<0$. The rest of the characteristic equation is considered in the form of

$$
\lambda^{3}+a_{1} \lambda^{2}+a_{2} \lambda+a_{3}=0
$$


where

$$
\begin{gathered}
a_{1}=\varepsilon+\theta+P \theta+2 d_{H}+d_{k}+d_{V}+\alpha N_{V} I_{V}^{*} \beta_{H}+N_{H} I_{H}^{*} \beta_{V} \\
a_{2}=d_{H}^{2}+(1-P) \varepsilon \theta+d_{V}(\varepsilon+\theta-P \theta)+a_{21}\left(\alpha \varepsilon+\alpha \theta+P \alpha \theta+\alpha \varepsilon \theta+P \alpha \varepsilon \theta+\alpha d_{V}\right) \\
+d_{k}\left(a_{22}\right)+N_{H} I_{H}^{*}\left(a_{22}\right) \beta_{V}+d_{H}\left(a_{22}+2\left(d_{k}+d_{V}+N_{H} I_{H}^{*} \beta_{V}\right)\right) \\
a_{3}=d_{k}\left(a_{31}-\alpha\left(\varepsilon+(P-1)(\varepsilon-1) \theta+d_{H}\right) a_{21}\right)+d_{V}\left(a_{31}-a_{32}\right) \\
+N_{H}\left(\alpha^{2} \varepsilon N_{V}^{2} S_{H}^{*}\left(I_{V}^{*}-1\right) I_{V}^{*} \beta_{H}^{*}+I_{H}^{*}\left(a_{31}-a_{32}\right)\right) \beta_{V} .
\end{gathered}
$$

where $a_{21}=N_{V} I_{V}^{*} \beta_{H}, a_{22}=\varepsilon+\theta-P \theta+\alpha a_{21}, a_{31}=\left((P-1) \theta-d_{H}\right)\left(\varepsilon+d_{H}\right)$, $a_{32}=\alpha\left(\varepsilon+(P-1)(\varepsilon-1) \theta+d_{H}\right) a_{21}$ and $S_{H}^{*}, I_{H}^{*}$, and $I_{V}^{*}$ are defined Equation (21).

By using Routh-Hurwitz criteria $[37,38]$ for $n=3$, then $E_{2}$ is stable if conditions $(i)-(i v)$ are satisfied. Since algebraic proofs may be a little difficult to verify these conditions, we then resort to numerical simulations. We plot the values of the conditions against the changes in $\beta_{H}$, which is shown in Figure 3. It is seen that these conditions are indeed satisfied (i) $a_{1}>0$, (ii) $a_{2}>0$, (iii) $a_{3}>0$, and (iv) $a_{1} a_{2}-a_{3}>0$.
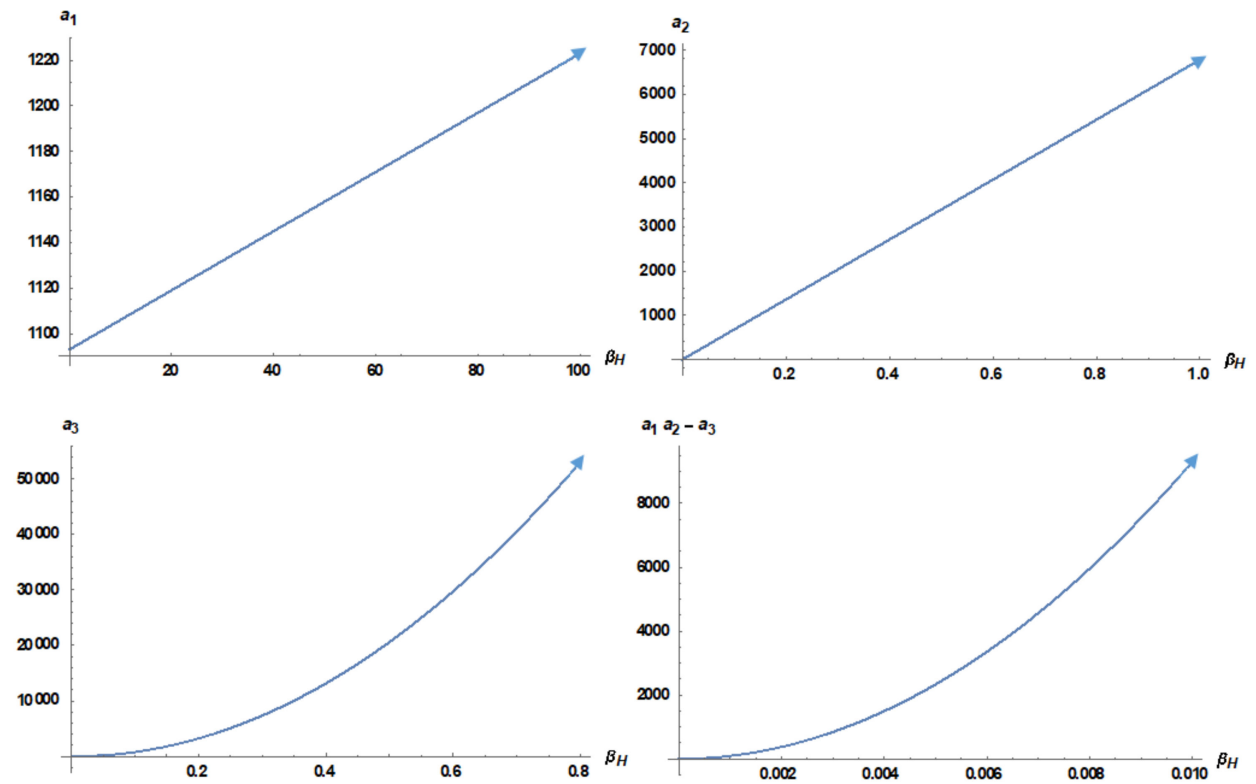

Figure 3. The Routh-Hurwitz criteria conditions satisfied $(i)-(i v)$ for the endemic equilibrium point.

It then follows that the polynomial of Equation (23) will indeed be Hurwitz, which thus implies the stability of the endemic equilibrium $E_{2}$.

\subsection{Global Stability Analysis}

The global stability of each equilibrium point of the model in system Equation (17) is investigated in this section. To serve this purpose, we hereby derive two theorems.

Definition 1. (Lyapunov) Let $x=0$ be an equilibrium point $\dot{x}=f(x)$ and $f(x): \phi \subset \mathbb{R}^{n}$. Let $V(x): \phi \rightarrow \mathbb{R}$ be a continuously differentiable function such that:

(i) $\quad V(0)=0$

(ii) $V(x)>0$ in $\phi$, except at $x=0$

(iii) $\dot{V}(0)=0$

(iv) $\dot{V}(x)<0$ in $\phi$, except at $x=0$

If $\dot{V}(x)<0$ in $\phi-\{0\}$, then $x=0$ is globally asymptotically stable. 
Theorem 3. Let $E_{1}^{*}=\left(S_{H}^{*}, E_{H}^{*}, I_{H}^{*}, S_{H}^{*}, I_{V}^{*}\right)=(1,0,0,0)$ the disease-free $E_{1}^{*}$ is globally asymptotically stable on $\phi$ if and only if $R_{0}<1$ where $\phi$ are defined as Proposition 1.

Proof. Consider the following Lyapunov function candidate [39-42]:

$$
\omega\left(S_{H}, E_{H}, I_{H}, I_{V}\right)=\left(S_{H}-S_{H}^{*} \ln S_{H}\right)+E_{H}+I_{H}+I_{V}
$$

This function satisfies the positive definiteness condition as required for a Lyapunov function since the positive invariances have been proven in Proposition 1. Furthermore, it should provide ease in the proving of the negative definiteness in its time derivative, as compared to say a quadratic candidate, which is more prominently used as one such candidate.

The derivative with respect to time yields:

$$
\begin{gathered}
\frac{d \omega}{d t}=\left(1-\frac{S_{H}^{*}}{S_{H}}\right) \frac{d S_{H}}{d t}+\frac{d E_{H}}{d t}+\frac{d I_{H}}{d t}+\frac{d I_{V}}{d t} \\
=\left(1-\frac{S_{H}^{*}}{S_{H}}\right)\left(b_{H}+(1-P)\left(1-S_{H}-E_{H}-I_{H}\right) \theta-\alpha \beta_{H} S_{H} I_{V} N_{V}-d_{H} S_{H}\right) \\
+\left(\alpha \beta_{H} S_{H} I_{V} N_{V}-\varepsilon E_{H}-d_{H} E_{H}\right)+\left(\varepsilon E_{H}-\gamma I_{H}-\left(d_{H}+d_{d}\right) I_{H}\right) \\
+\left(\beta_{V}\left(1-I_{V}\right) I_{H} N_{H}-\left(d_{V}+d_{k}\right) I_{V}\right) \\
=\left(1-\frac{S_{H}^{*}}{S_{H}}\right)\left(b_{H}+(1-P)\left(1-S_{H}-E_{H}-I_{H}\right) \theta-\alpha \beta_{H} S_{H} I_{V} N_{V}-d_{H} S_{H}\right) \\
-d_{H} E_{H}-\left(d_{H}+d_{d}\right) I_{H}+\left(\beta_{V}\left(1-I_{V}\right) I_{H} N_{H}-\left(d_{V}+d_{k}\right) I_{V}\right)
\end{gathered}
$$

Substituting the relation of disease-free $E_{1}^{*}=(1,0,0,0)$, we obtain:

$$
\begin{gathered}
=\left(1-\frac{S_{H}^{*}}{S_{H}}\right)\left(b_{H}-d_{H} S_{H}\right)-d_{H} E_{H}-\left(d_{H}+d_{d}\right) I_{H}-\left(d_{V}+d_{k}\right) I_{V} \\
=\left[d_{H}\left(1-\frac{S_{H}}{S_{H}^{*}}\right)+b_{H}\left(1-\frac{S_{H}^{*}}{S_{H}}\right)\right]-d_{H} E_{H}-\left(d_{H}+d_{d}\right) I_{H}-\left(d_{V}+d_{k}\right) I_{V}
\end{gathered}
$$

From Equation (11) at the disease-free, we obtain $b_{H}=d_{H}$ thus

$$
\begin{gathered}
=\left[d_{H}\left(1-\frac{S_{H}}{S_{H}^{*}}\right)+d_{H}\left(1-\frac{S_{H}^{*}}{S_{H}}\right)\right]-d_{H} E_{H}-\left(d_{H}+d_{d}\right) I_{H}-\left(d_{V}+d_{k}\right) I_{V} \\
=d_{H}\left(2-\frac{S_{H}}{S_{H}^{*}}-\frac{S_{H}^{*}}{S_{H}}\right)-d_{H} E_{H}-\left(d_{H}+d_{d}\right) I_{H}-\left(d_{V}+d_{k}\right) I_{V} \\
=-d_{H}\left(\frac{\left(S_{H}^{*}-S_{H}\right)^{2}}{S_{H}^{*} S_{H}}\right)-d_{H} E_{H}-\left(d_{H}+d_{d}\right) I_{H}-\left(d_{V}+d_{k}\right) I_{V} \\
\frac{d \omega}{d t}=-\left[d_{H}\left(\frac{\left(S_{H}^{*}-S_{H}\right)^{2}}{S_{H}^{*} S_{H}}\right)+d_{H} E_{H}+\left(d_{H}+d_{d}\right) I_{H}+\left(d_{V}+d_{k}\right) I_{V}\right]
\end{gathered}
$$

As can be seen, all of the terms in Equation (24) are always negative. Now, using LaSalle's invariance principle [39], we have $\frac{d \omega}{d t} \leq 0$ and so the function $\frac{d \omega}{d t}$ is to be negative definite. Each solution's limit set is included in the biggest invariant set for which $S_{H}=S_{H}^{*}, E_{H}=E_{H}^{*}, I_{H}=I_{H}^{*}$, and $I_{V}=I_{V}^{*}$ which is the singleton $\left\{E_{1}^{*}\right\}$. The disease-free equilibrium point $E_{1}^{*}$ is then globally asymptotically stable on $\phi$ according to LaSalle's invariant principle. 
Theorem 4. When $R_{0}>1$, the endemic equilibrium point $E_{2}^{*}$ is defined as Equation (18). Then Equation (21) is globally asymptotically stable on $\phi$ if and only if

$$
\left\{\begin{array}{l}
b_{H}=d_{H} S_{H}^{*} \\
I_{V}=\frac{\beta_{V} I_{H} N_{H}}{\beta_{V} I_{H} N_{H}+d_{V}+d_{k}}
\end{array}\right.
$$

Proof. The Lyapunov function is in the form:

$$
\xi\left(S_{H}, E_{H}, I_{H}, I_{V}\right)=\left(S_{H}-S_{H}^{*} \ln S_{H}\right)+E_{H}+I_{H}+I_{V}
$$

The derivative with respect to time yields:

$$
\begin{gathered}
\frac{d \xi}{d t}=\left(1-\frac{S_{H}^{*}}{S_{H}}\right) \frac{d S_{H}}{d t}+\frac{d E_{H}}{d t}+\frac{d I_{H}}{d t}+\frac{d I_{V}}{d t} \\
=\left(1-\frac{S_{H}^{*}}{S_{H}}\right)\left(b_{H}+(1-P)\left(1-S_{H}-E_{H}-I_{H}\right) \theta-\alpha \beta_{H} S_{H} I_{V} N_{V}-d_{H} S_{H}\right) \\
-d_{H} E_{H}-\left(d_{H}+d_{d}\right) I_{H}+\left(\beta_{V}\left(1-I_{V}\right) I_{H} N_{H}-\left(d_{V}+d_{k}\right) I_{V}\right) \\
=\left(\begin{array}{c}
b_{H}-b_{H} \frac{S_{H}^{*}}{S_{H}}-d_{H} S_{H}+d_{H} S_{H}^{*}+(1-P)\left(1-S_{H}-E_{H}-I_{H}\right) \theta \\
-(1-P)\left(1-S_{H}-E_{H}-I_{H}\right) \theta_{H}^{*}
\end{array}\right)-d_{H} E_{H}-\left(d_{H}+d_{d}\right) I_{H} \\
+\left(\beta_{V}\left(1-I_{V}\right) I_{H} N_{H}-\left(d_{V}+d_{k}\right) I_{V}\right) \\
=\left(b_{H}\left(1-\frac{S_{H}^{*}}{S_{H}}\right)+d_{H} S_{H}^{*}\left(1-\frac{S_{H}}{S_{H}^{*}}\right)+(1-P)\left(1-S_{H}-E_{H}-I_{H}\right) \theta\left(1-\frac{S_{H}^{*}}{S_{H}}\right)\right) \\
-d_{H} E_{H}-\left(d_{H}+d_{d}\right) I_{H}+\left(\beta_{V}\left(1-I_{V}\right) I_{H} N_{H}-\left(d_{V}+d_{k}\right) I_{V}\right)
\end{gathered}
$$

Substituting the expressions for $d_{H}$ and $I_{V}$, we obtain:

$$
\begin{gathered}
=b_{H}\left(\left(1-\frac{S_{H}^{*}}{S_{H}}\right)+\left(1-\frac{S_{H}}{S_{H}^{*}}\right)\right)-d_{H} E_{H}-\left(d_{H}+d_{d}\right) I_{H} \\
=-b_{H}\left(\frac{\left(S_{H}^{*}-S_{H}\right)^{2}}{S_{H}^{*} S_{H}}\right)-d_{H} E_{H}-\left(d_{H}+d_{d}\right) I_{H} \\
\frac{d \xi}{d t}=-\left(b_{H} \frac{\left(S_{H}^{*}-S_{H}\right)^{2}}{S_{H}^{*} S_{H}}+d_{H} E_{H}+\left(d_{H}+d_{d}\right) I_{H}\right)
\end{gathered}
$$

Hence, the condition of Equation (26) shows that $\frac{d \tilde{\xi}}{d t} \leq 0$ for $\left(S_{H}, E_{H}, I_{H}, I_{V}\right) \in \phi$, and the strict quality holds only for $S_{H}=S_{H}^{*}, E_{H}=E_{H}^{*}, I_{H}=I_{H}^{*}$, and $I_{V}=I_{V}^{*}$. Then the endemic equilibrium point $E_{2}^{*}$ is globally asymptotically stable in $\phi$.

Note that the exact solution of the system may also be revived by using the powerful tool of Lie algebra. For further information, see the works of Shang $[43,44]$.

\section{Numerical Simulation}

In this section, we investigate the impact of the transmission of dengue consider only re-infectious, regardless of individual serotypes each time they were infected. The initial values for the parameter are listed in Table 2 . The parameter values of dengue cases in Thailand are estimated. The information was gathered from the Ministry of Public Health's Department of Disease Control. Note that although most parameter's initial values are taken from the source literature. However, certain values must be estimated and assumed for the purposes of this model. There are four parameters to be estimated: $N_{H}, N_{V}, d_{d}$, and $d_{k}$ and two parameters to be assumed: $\beta_{H}$ and $\beta_{V}$. The model of system Equation (17) is 
then simulated with the use of a differential equation solver in MATLAB. The results of our present numerical simulation are shown in Figures 4-7.

Table 2. The initial value for the parameters.

\begin{tabular}{cccc}
\hline Parameters & The Disease-Free & The Endemic & Source \\
\hline$\alpha$ & $1 / 7$ & $1 / 7$ & {$[45-50]$} \\
$\varepsilon$ & $1 / 10$ & $1 / 10$ & {$[45-50]$} \\
$P$ & 0.5 & 0.5 & {$[45-50]$} \\
$\theta$ & $1 /(30 \times 6)$ & $1 /(30 \times 6)$ & {$[45-50]$} \\
$N_{H}$ & 250,000 & 250,000 & estimated \\
$N_{V}$ & 200,000 & 200,000 & estimated \\
$\beta_{H}$ & 0.00000025 & 0.005 & assumed \\
$\beta_{V}$ & 0.00000012 & 0.003 & assumed \\
$b_{H}$ & $1 /(365 \times 70)$ & $1 /(365 \times 70)$ & {$[45-50]$} \\
$d_{H}$ & $1 /(365 \times 70)$ & $1 /(365 \times 70)$ & {$[45-50]$} \\
$d_{V}$ & $1 / 14$ & $1 / 14$ & {$[45-50]$} \\
$d_{d}$ & $1 / 14$ & $1 / 14$ & estimated \\
$d_{k}$ & $1 / 7$ & $1 / 7$ & estimated \\
$\gamma$ & $1 / 14$ & $1 / 14$ & {$[45-50]$} \\
\hline
\end{tabular}

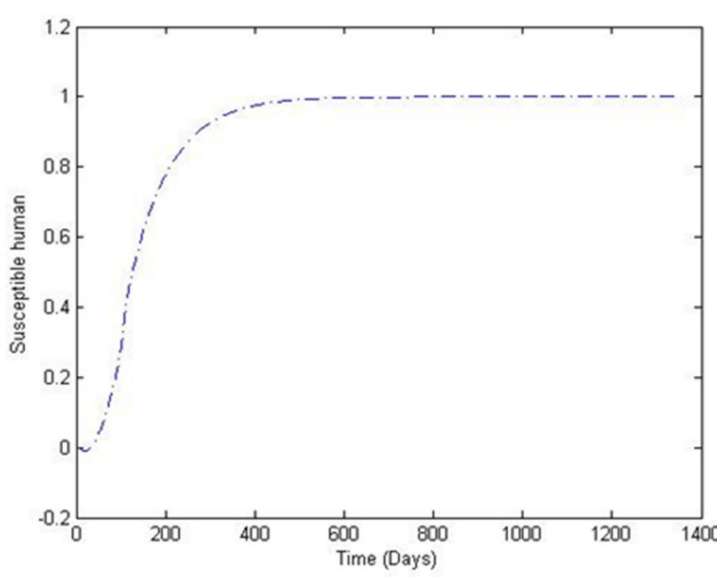

(a)

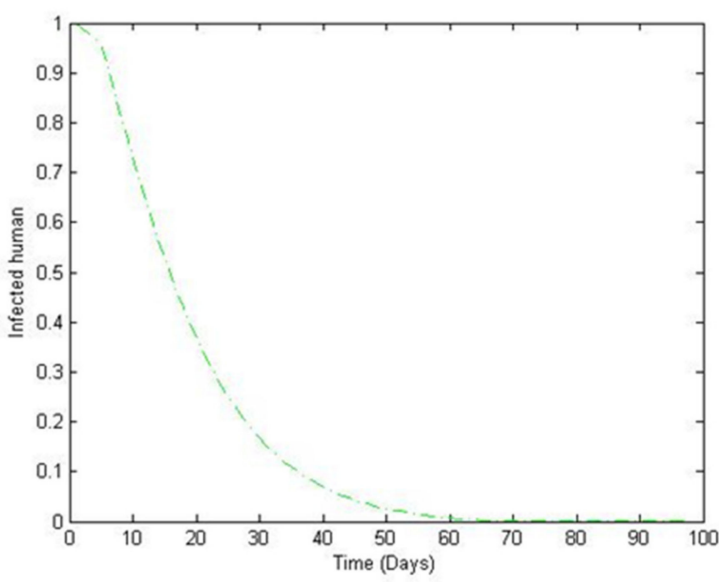

(c)

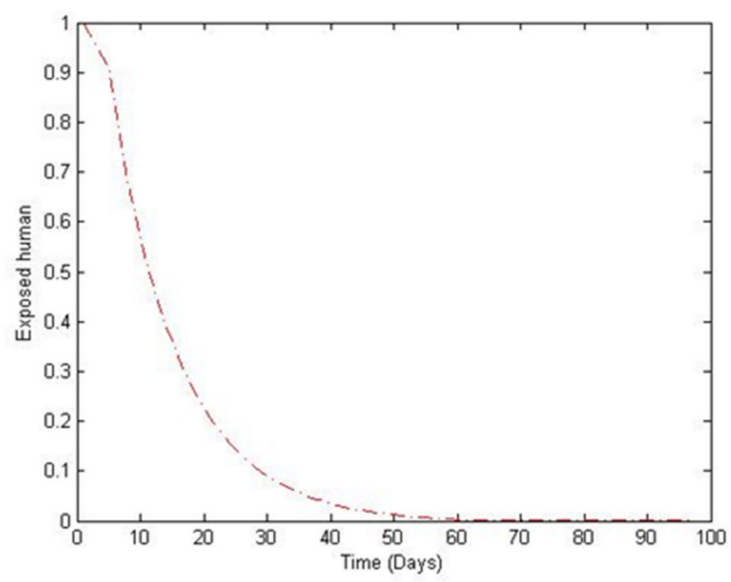

(b)

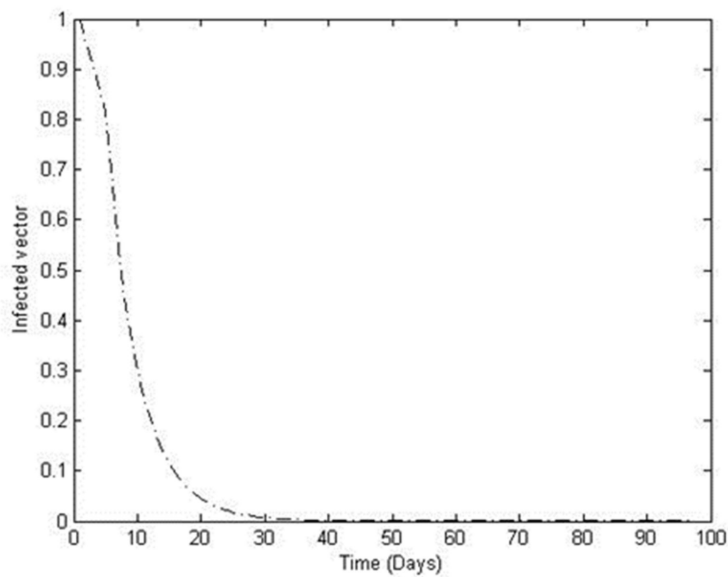

(d)

Figure 4. Graphs of system Equation (17) at the disease-free equilibrium point of $S_{H}, E_{H}, I_{H}$, and $I_{V}$ for $R_{0}<1$. (a) Susceptible human, (b) exposed human, (c) infected human, and (d) infected vector. 


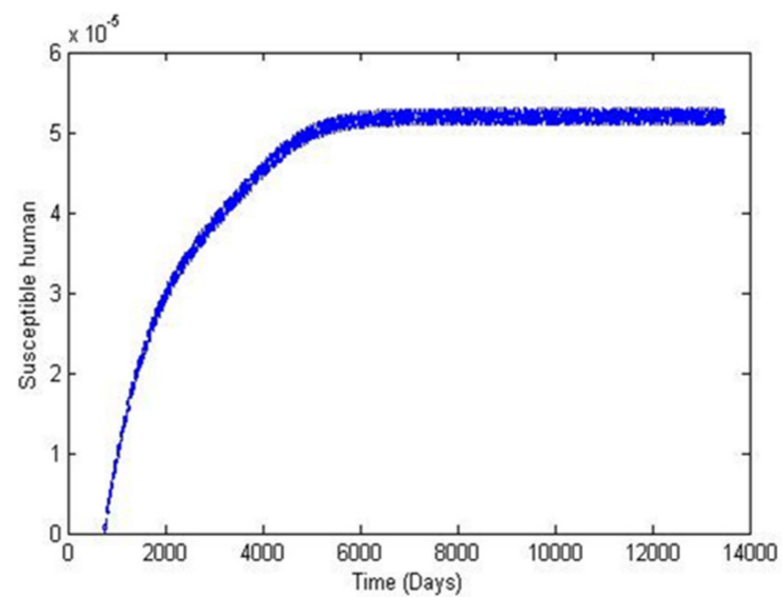

(a)

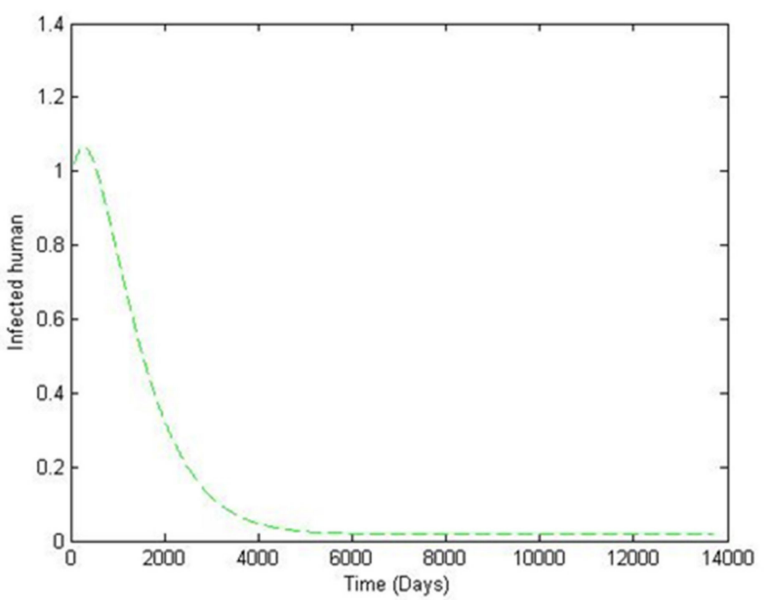

(c)

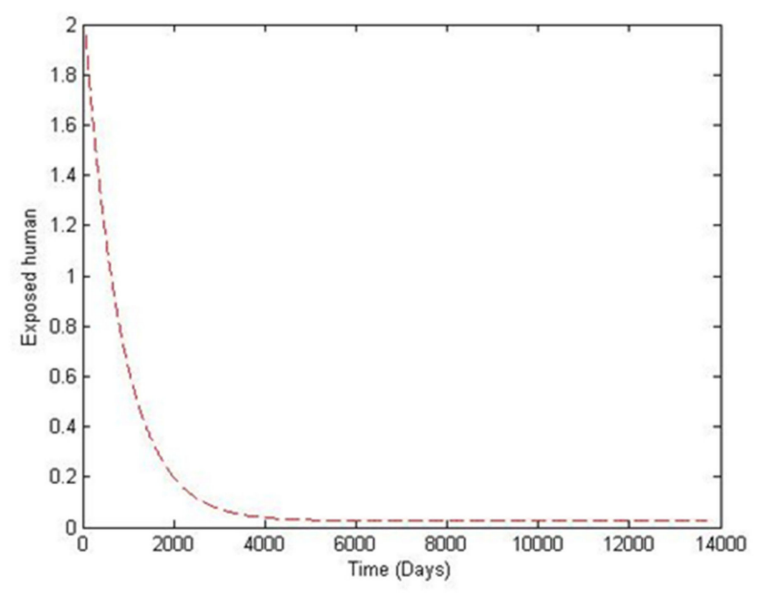

(b)

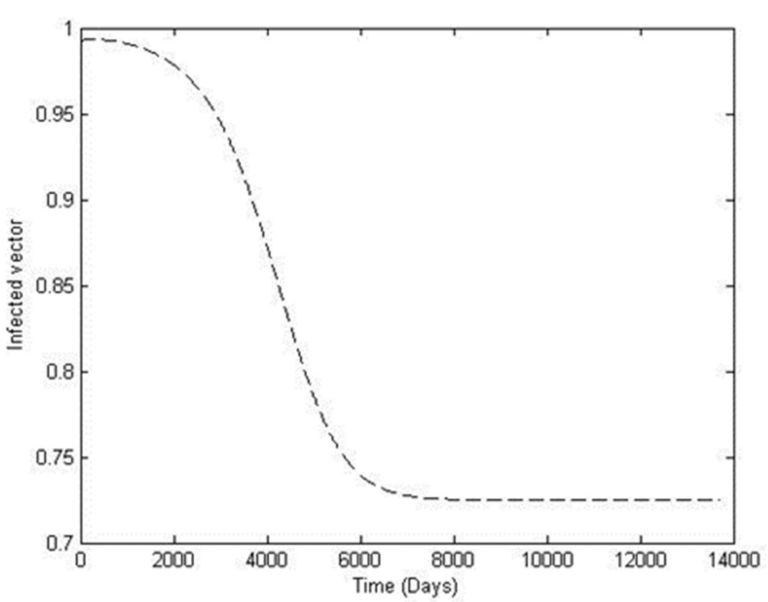

(d)

Figure 5. Graphs of system Equation (17) at the endemic equilibrium point of $S_{H}, E_{H}, I_{H}$, and $I_{V}$ for $R_{0}>1$. (a) Susceptible human, (b) exposed human, (c) infected human, and (d) infected vector.

The model of Equation (17) is solved using the differential solver ode45 in MATLAB. This solver employs the fourth-order Runge-Kutta method with adjustable step size to solve the system of differential equations.

Figure 4 shows the numerical solution of the disease-free equilibrium where $R_{0}<1$ after about 500,60, 65, and 30 days for the susceptible human, exposed human, infected human, and infected vector it converges to $E_{1}=(1,0,0,0)$, respectively. Figure 5 shows the numerical solution of the endemic equilibrium where $R_{0}>1$ after about 6500,4000, 5000, and 7000 days for the susceptible human, exposed human, infected human, and infected vector it converges to $E_{2}=(0.00005,0.02689,0.01881,0.73048)$, respectively. Figures 6 and 7 show the numerical solution of the endemic equilibrium with compares parameters the dengue virus transmission rate from vector to human $\beta_{H}=0.01,0.03,0.05,0.07,0.09$ and the dengue virus transmission rate from human to vector $\beta_{V}=0.002,0.004,0.006,0.008,0.010$ for the susceptible human, exposed human, infected human, and infected vector. We can observe that the higher the dengue virus transmission rate from vector to human $\beta_{H}$ and the dengue virus transmission rate from human to vector $\beta_{V}$, the slower the convergence to a susceptible human equilibrium point. The exposed human, infected human, and infected vector all rapidly reach a point of equilibrium, respectively. 


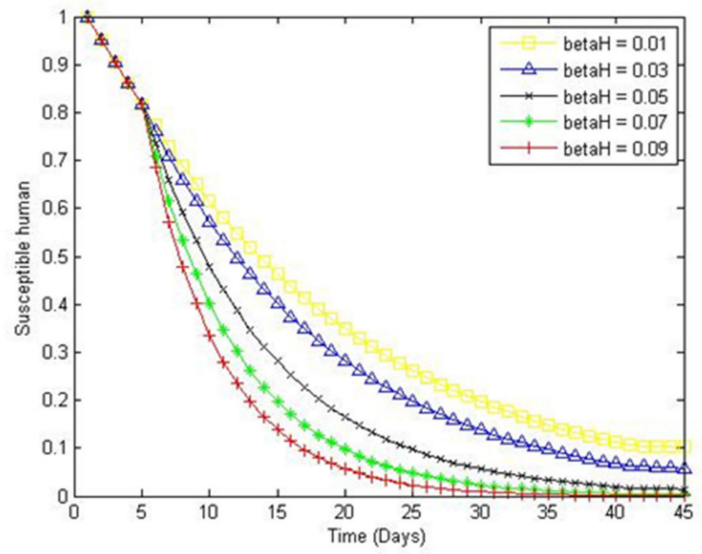

(a)

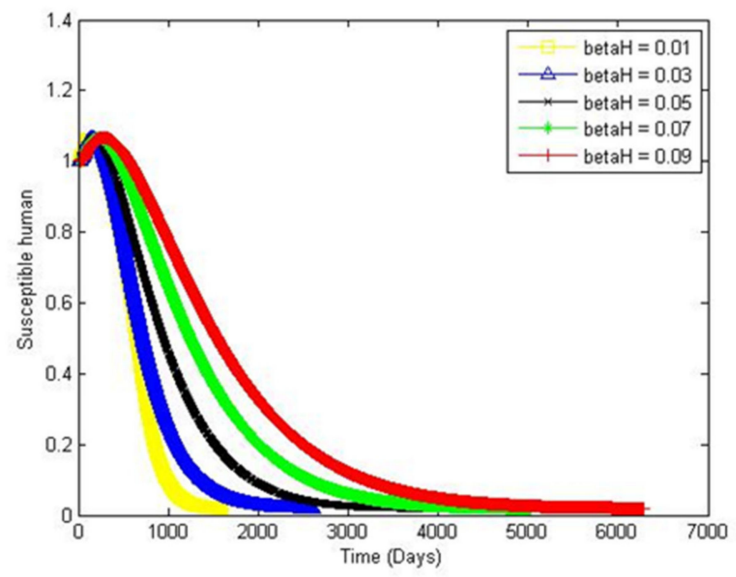

(c)

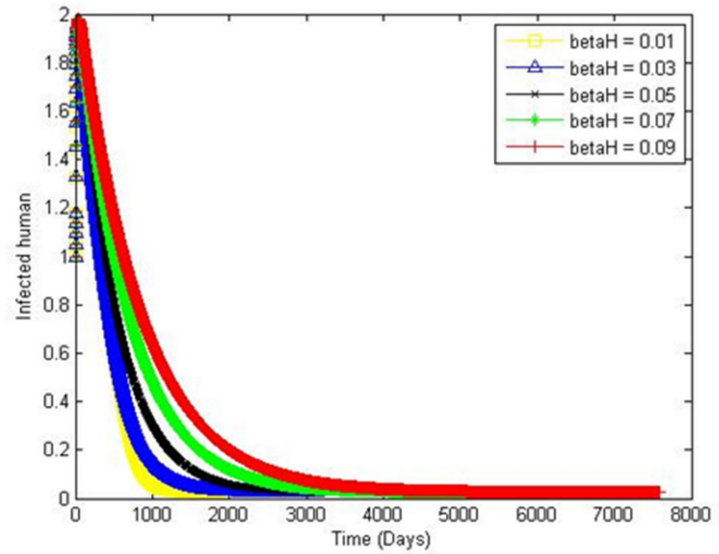

(b)

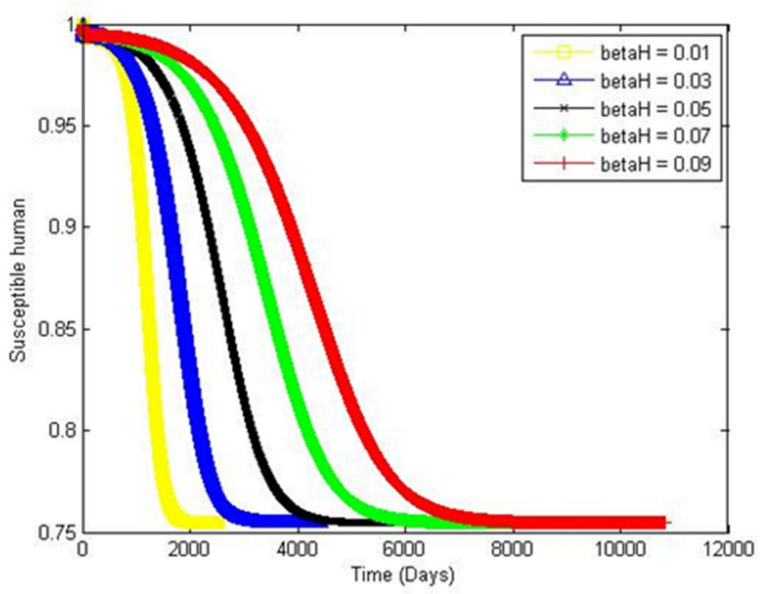

(d)

Figure 6. Graphs of system Equation (17) at the endemic equilibrium point of $S_{H}, E_{H}, I_{H}$, and $I_{V}$ for $R_{0}>1$ with compares parameter $\beta_{H}=0.01,0.03,0.05,0.07,0.09$. (a) Susceptible human, (b) exposed human, (c) infected human, and (d) infected vector. 


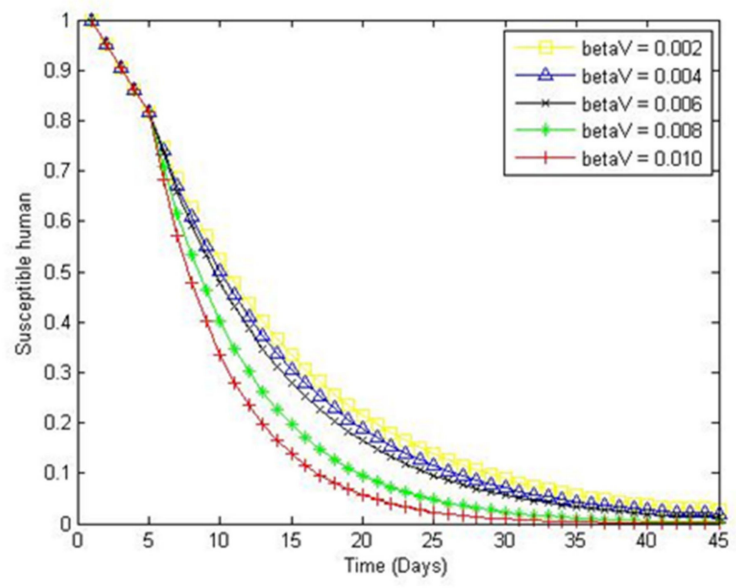

(a)

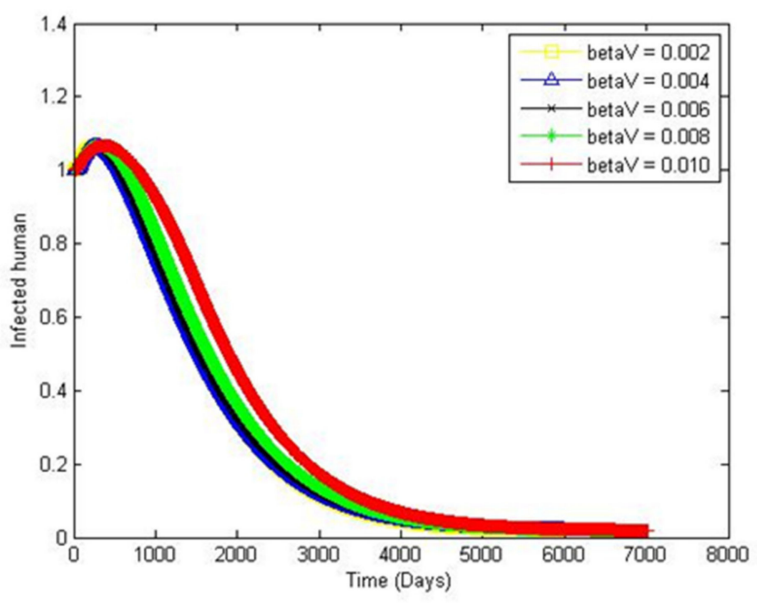

(c)

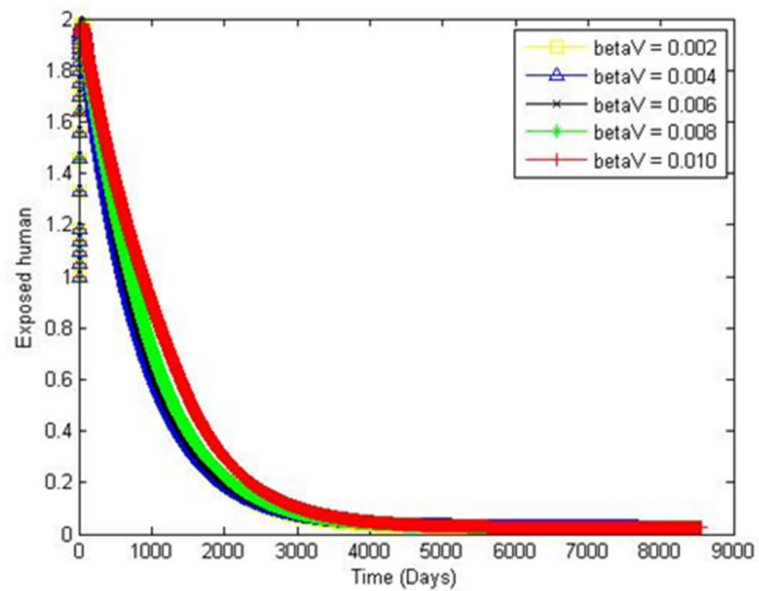

(b)

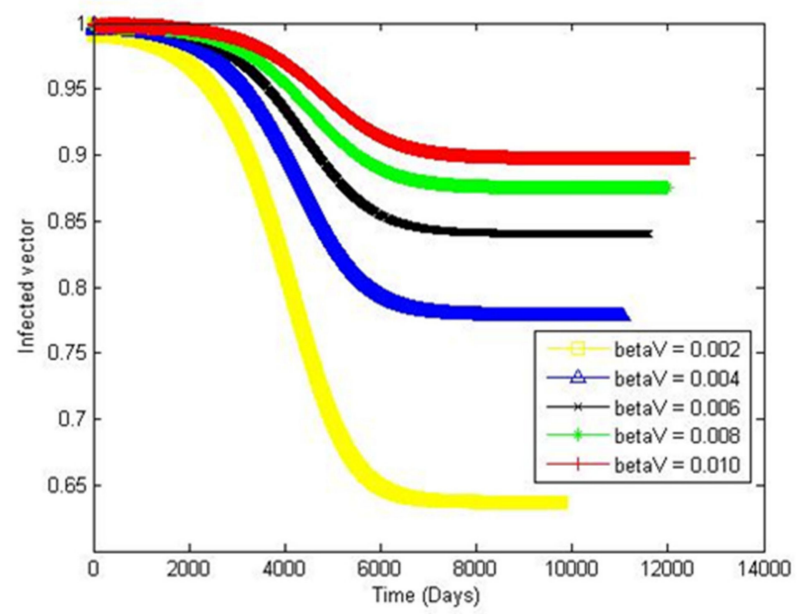

(d)

Figure 7. Graphs of system Equation (17) at the endemic equilibrium point of $S_{H}, E_{H}, I_{H}$, and $I_{V}$ for $R_{0}>1$ with compares parameter $\beta_{V}=0.002,0.004,0.006,0.008,0.010$. (a) Susceptible human, (b) exposed human, (c) infected human, and (d) infected vector.

\section{Optimal Control Strategies}

In this section, we apply the Pontryagin's maximum principle (PMP) to derive the necessary conditions for the solution for the existence of optimal control. Equations (17) can be rewritten as an optimal control problem with the goal of reducing the number of infected human populations. Two control inputs may be assigned to the system since they include the dynamics of both human and vector populations, namely $u_{1}$ where this control input represents an effort to reduce the number of infected humans by giving vaccinations to humans, and $u_{2}$ for the vector population represents the administration of repellents to mosquitoes and destroying mosquito breeding sites. The system of equations with control is written as:

$$
\begin{gathered}
\frac{d S_{H}}{d t}=b_{H}+(1-P) R_{H} \theta-\alpha \beta_{H} S_{H} I_{V} N_{V}-d_{H} S_{H}-u_{1}(t) I_{H} \\
\frac{d E_{H}}{d t}=\alpha \beta_{H} S_{H} I_{V} N_{V}-\varepsilon E_{H}-d_{H} E_{H} \\
\frac{d I_{H}}{d t}=\varepsilon E_{H}-\gamma I_{H}-\left(d_{H}+d_{d}\right) I_{H}
\end{gathered}
$$




$$
\begin{gathered}
\frac{d R_{H}}{d t}=\gamma I_{H}-(1-P) \theta R_{H}-d_{H} R_{H} \\
\frac{d S_{V}}{d t}=\frac{A}{N_{V}}-\beta_{V} S_{V} I_{H} N_{H}-d_{V} S_{V}-u_{2}(t) S_{V} \\
\frac{d I_{V}}{d t}=\beta_{V} S_{V} I_{H} N_{H}-\left(d_{V}+d_{k}\right) I_{V}-u_{2}(t) \beta_{V} S_{V} I_{H} N_{H}
\end{gathered}
$$

In normalized compartments Equations (27)-(32) are:

$$
\begin{gathered}
\frac{d S_{H}}{d t}=b_{H}+(1-P)\left(1-S_{H}-E_{H}-I_{H}\right) \theta-\alpha \beta_{H} S_{H} I_{V} N_{V}-d_{H} S_{H}-u_{1}(t) I_{H} \\
\frac{d E_{H}}{d t}=\alpha \beta_{H} S_{H} I_{V} N_{V}-\left(\varepsilon+d_{H}\right) E_{H} \\
\frac{d I_{H}}{d t}=\varepsilon E_{H}-\left(d_{H}+d_{d}+\gamma\right) I_{H} \\
\frac{d I_{V}}{d t}=\left(1-u_{2}(t)\right) \beta_{V} I_{H} N_{H}\left(1-I_{V}\right)-\left(d_{V}+d_{k}\right) I_{V}
\end{gathered}
$$

All parameter definitions retain their meanings from the uncontrolled system. For non-negative bounded initial conditions, this system has non-negative bounded solution (Lukes 1982 [51]). The objective function to be minimized is written:

$$
J(u)=\int_{0}^{T}\left[C_{1} I_{H}+C_{2} I_{V}+\frac{1}{2}\left(D_{1} u_{1}^{2}(t)+D_{2} u_{2}^{2}(t)\right)\right] d t
$$

with initial condition $S_{H}, E_{H}, I_{V} \geq 0$, and $I_{H} \geq 0$. The weight constants are $C_{1}$ and $C_{2}$ for the number of infected humans and the infected vector populations. The weights $D_{1}$ and $D_{2}$ are measures of the costs associated with the control variables $u_{1}$ and $u_{2}$ respectively. The Hamiltonian for the Lagrangian problem of the optimal control problem and the optimal control problem by Lagrangian was defined as follows:

$$
L=C_{1} I_{H}+C_{2} I_{V}+\frac{1}{2}\left(D_{1} u_{1}^{2}(t)+D_{2} u_{2}^{2}(t)\right)
$$

Theorem 5. There exists an optimal control $u_{1}^{*}(t)$ and $u_{2}^{*}(t)$ such that $\left.J\left(u_{1}^{*}, u_{2}^{*}\right)=\min \left\{J\left(u_{1}, u_{2}\right) \mid\left(u_{1}, u_{2}\right) \in U\right)\right\}$.

Proof. The existence of the optimal control problem Equations (33)-(36) we apply results from $[48,49]$ to prove the analyzed existence results of the optimal control problem.

The existence of the system in Equations (33)-(36) is given which is non-empty and bounded, according to Theorem 9.2.1 from Lukes [51]. The control set $U$ is closed and convex. The right side of the control system Equations (33)-(36) is linear in $u_{1}$ and $u_{2}$. The integrand $L$ is convex on $U$. To prove the bound on $L$, let $m_{2}=\min \left(I_{H}(t), I_{V}(t)\right)$, $m_{1}=\left(D_{1}, D_{2}\right)$, and $\psi=2$. Then the Lagrangian function $L$ defined as:

$$
\begin{gathered}
L\left(I_{H}, I_{V}, u_{1}, u_{2}\right)=C_{1} I_{H}+C_{2} I_{V}+\frac{1}{2}\left(D_{1} u_{1}^{2}(t)+D_{2} u_{2}^{2}(t)\right) \\
\geq m_{2}\left(I_{H}+I_{V}\right)+m_{1}\left(\left|u_{1}\right|^{2}+\left|u_{2}\right|^{2}\right) \\
=m_{2}+m_{1}\left(\left|u_{1}\right|^{2}+\left|u_{2}\right|^{2}\right)
\end{gathered}
$$

where $m_{1}, m_{2}, C_{1}, C_{2}, D_{1}, D_{2}>0$, and $\psi>1$. Therefore, there exists an optimal control for the system of Equations (33)-(36) satisfying the Pontryagin's minimum principle $[25-29,51,52]$. 
Theorem 6. Let $u_{1}^{*}$ and $u_{1}^{*}$ be optimal controls, and let $S_{H}, E_{H}, I_{H}$, and $I_{V}$ be the solutions of the optimal control problem Equations (33)-(36) that minimize $J\left(u_{1}, u_{2}\right) \in U$. Then there are adjoint variables $\lambda_{1}=\lambda_{S_{H}}, \lambda_{2}=\lambda_{E_{H}}, \lambda_{3}=\lambda_{I_{H}}$, and $\lambda_{4}=\lambda_{I_{V}}$ satisfying the adjoint system of equations:

$$
\begin{gathered}
\frac{d \lambda_{1}}{d t}=-\lambda_{1}(t)\left((P-1) \theta-\alpha \beta_{H} I_{V} N_{V}-d_{H}\right)-\lambda_{2}\left(\alpha \beta_{H} I_{V} N_{V}\right) \\
\frac{d \lambda_{2}}{d t}=-\lambda_{1}(t)(P-1) \theta-\lambda_{2}\left(-\varepsilon-d_{H}\right)-\lambda_{3}(\varepsilon) \\
\frac{d \lambda_{3}}{d t}=-\lambda_{1}(t)\left((P-1) \theta-u_{1}^{*}(t)\right)-\lambda_{3}\left(-d_{H}-d_{d}-\gamma\right)-\lambda_{4}\left(\left(1-u_{2}^{*}(t)\right) \beta_{V} N_{H}\left(1-I_{V}\right)\right)-C_{1} \\
\frac{d \lambda_{4}}{d t}=-\lambda_{1}(t)\left(-\alpha \beta_{H} S_{H} N_{V}\right)-\lambda_{2}\left(\alpha \beta_{H} S_{H} N_{V}\right)-\lambda_{4}\left(-\beta_{V} I_{H} N_{H}+u_{2}^{*}(t) I_{H} \beta_{V} N_{H}-d_{V}-d_{k}\right)-C_{2}
\end{gathered}
$$

where $S_{H}, E_{H}, I_{H}$, and $I_{V}$ are the adjoint variables, and the controls $u_{1}^{*}$ and $u_{2}^{*}$ obey the optimality conditions

$$
\begin{gathered}
u_{1}^{*}(t)=\max \left(\min \left(\frac{\lambda_{1} I_{H}^{*}}{D_{1}}, u_{1}^{\max }\right), 0\right), \\
u_{2}^{*}(t)=\max \left(\min \left(\frac{\left(1-I_{V}^{*}\right) \beta_{V} I_{H}^{*} N_{H}}{D_{2}}, u_{2}^{\max }\right), 0\right) .
\end{gathered}
$$

Proof. The corresponding Hamiltonian is defined as:

$$
\begin{gathered}
H=L\left(I_{H}, I_{V}, u_{1}, u_{2}\right)+\lambda_{1} \frac{d S_{H}}{d t}++\lambda_{2} \frac{d E_{H}}{d t}+\lambda_{3} \frac{d I_{H}}{d t}+\lambda_{4} \frac{d I_{V}}{d t} \\
H=C_{1} I_{H}+C_{2} I_{V}+\frac{1}{2}\left(D_{1} u_{1}^{2}(t)+D_{2} u_{2}^{2}(t)\right) \\
+\lambda_{1}\left[b_{H}+(1-P)\left(1-S_{H}-E_{H}-I_{H}\right) \theta-\alpha \beta_{H} S_{H} I_{V} N_{V}-d_{H} S_{H}-u_{1}(t) I_{H}\right] \\
+\lambda_{2}\left[\alpha \beta_{H} S_{H} I_{V} N_{V}-\left(\varepsilon+d_{H}\right) E_{H}\right]+\lambda_{3}\left[\varepsilon E_{H}-\left(d_{H}+d_{d}+\gamma\right) I_{H}\right] \\
+\lambda_{4}\left[\left(1-u_{2}(t)\right) \beta_{V} I_{H} N_{H}\left(1-I_{V}\right)-\left(d_{V}+d_{k}\right) I_{V}\right]
\end{gathered}
$$

The associated adjoint system is as follows:

$$
\begin{gathered}
\frac{d \lambda_{1}}{d t}=-\frac{\partial H}{\partial S_{H}}=-\lambda_{1}(t)\left((P-1) \theta-\alpha \beta_{H} I_{V} N_{V}-d_{H}\right)-\lambda_{2}\left(\alpha \beta_{H} I_{V} N_{V}\right) \\
\frac{d \lambda_{2}}{d t}=-\frac{\partial H}{\partial E_{H}}=-\lambda_{1}(t)(P-1) \theta-\lambda_{2}\left(-\varepsilon-d_{H}\right) \\
\frac{d \lambda_{3}}{d t}=-\frac{\partial H}{\partial I_{H}}=-\lambda_{1}(t)\left((P-1) \theta-u_{1}^{*}(t)\right)-\lambda_{3}\left(-d_{H}-d_{d}-\gamma\right) \\
-\lambda_{4}\left(\left(1-u_{2}^{*}(t)\right) \beta_{V} N_{H}\left(1-I_{V}\right)\right)-C_{1} \\
\frac{d \lambda_{4}}{d t}=-\frac{\partial H}{\partial I_{V}}=-\lambda_{1}(t)\left(-\alpha \beta_{H} S_{H} N_{V}\right)-\lambda_{2}\left(\alpha \beta_{H} S_{H} N_{V}\right) \\
-\lambda_{4}\left(-\beta_{V} I_{H} N_{H}+u_{2}^{*}(t) I_{H} \beta_{V} N_{H}-d_{V}-d_{k}\right)-C_{2}
\end{gathered}
$$

with the boundary conditions

$$
\lambda_{1}(t)=0, \lambda_{2}(t)=0, \lambda_{3}(t)=0, \lambda_{4}(t)=0
$$

Using the properties of optimal set optimality conditions, we find that

$$
\frac{\partial H}{\partial u_{1}}=\frac{\partial H}{\partial u_{2}}=0 \text { at } u_{1}=u_{1}^{*} \text { and } u_{2}=u_{2}^{*}
$$

Therefore,

$$
\begin{gathered}
\frac{\partial H}{\partial u_{1}}=D_{1} u_{1}-\lambda_{1} I_{H}=0 \\
u_{1}^{*}=\frac{\lambda_{1} I_{H}}{D_{1}} \\
\frac{\partial H}{\partial u_{2}}=D_{2} u_{2}-\left(1-I_{V}\right) \beta_{V} I_{H} N_{H}=0 \\
u_{2}^{*}=\frac{\left(1-I_{V}\right) \beta_{V} I_{H} N_{H}}{D_{2}}
\end{gathered}
$$


The optimal controls $u_{1}^{*}$ and $u_{2}^{*}$ are then given by:

$$
\begin{gathered}
u_{2}^{*}= \begin{cases}0 & ; \frac{\lambda_{1} I_{H}}{D_{1}} \leq 0, \\
\frac{\lambda_{1} I_{H}}{D_{1}} & ; \frac{\lambda_{1} I_{H}}{D_{1}}<u_{1}^{\max } \\
u_{1}^{\max } & ; \frac{\lambda_{1} I_{H}}{D_{1}} \geq u 1 \mathrm{max} .\end{cases} \\
u_{2}^{*}= \begin{cases}0 & ; \frac{\left(1-I_{V}\right) \beta_{V} I_{H} N_{H}}{D_{2}} \leq 0, \\
\frac{\left(1-I_{V}\right) \beta_{V} I_{H} N_{H}}{D_{2}} & ; \frac{\left(1-I_{V}\right) \beta_{V} I_{H} N_{H}}{D_{2}}<u_{2}^{\max }, \\
u_{2}^{\max } & ; \frac{\left(1-I_{V}\right) \beta_{V} I_{H} N_{H}}{D_{2}} \geq u_{2}^{\max } .\end{cases}
\end{gathered}
$$

The fourth-order Runge-Kutta method, in conjunction with the forward-backward method, is used to solve the optimal control problem numerically. The solution results for optimal strategies control are shown in Figures 8-13 the initial values parameter according to listed in Table 2.

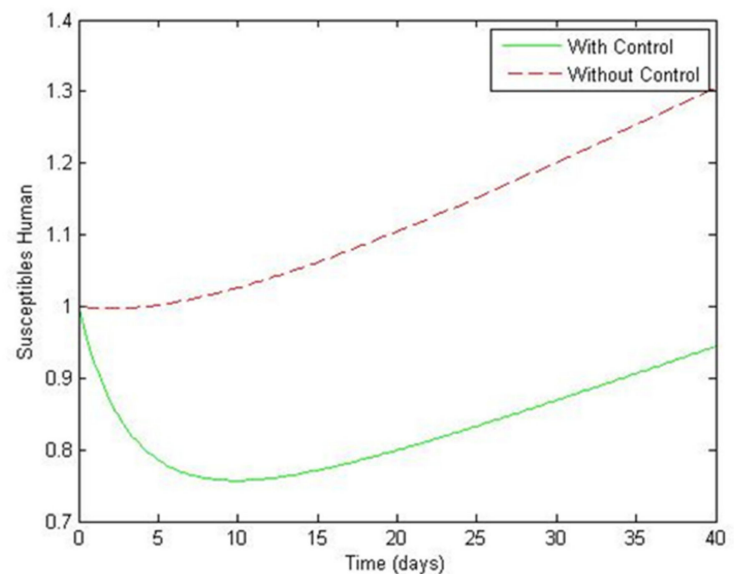

(a)

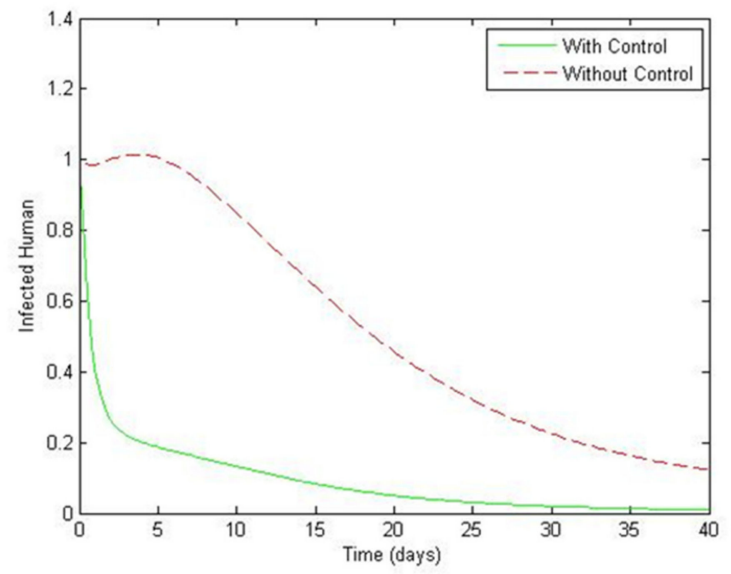

(c)

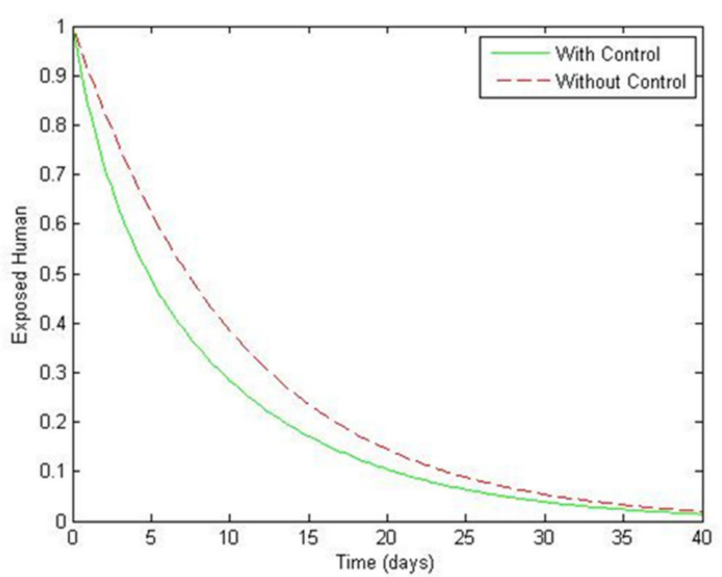

(b)

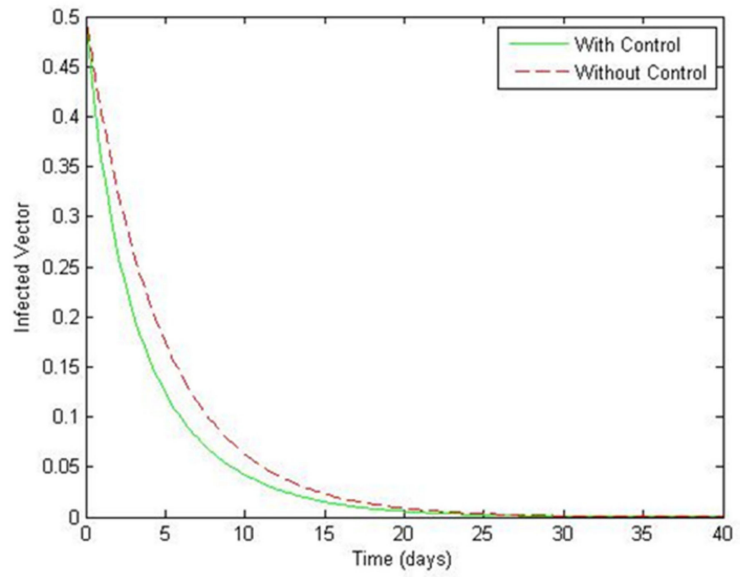

(d)

Figure 8. Comparison of behaviors with and without of system of Equations (33)-(36) of $S_{H}, E_{H}, I_{H}$, and $I_{V}$ when using $C_{1}=0.000005, C_{2}=50$. (a) Susceptible human (b) exposed human (c) infected human and (d) infected vector. 


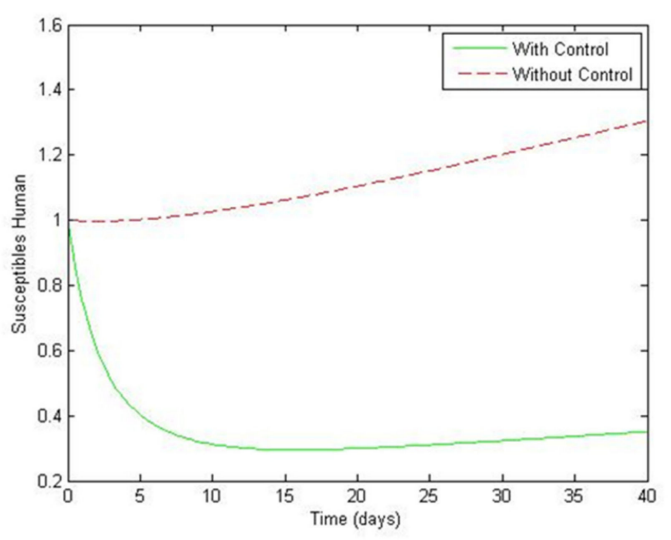

(a)

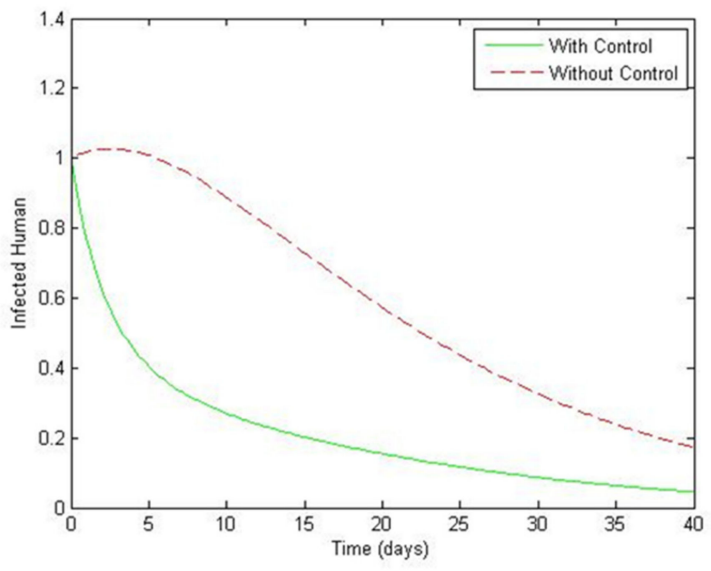

(c)

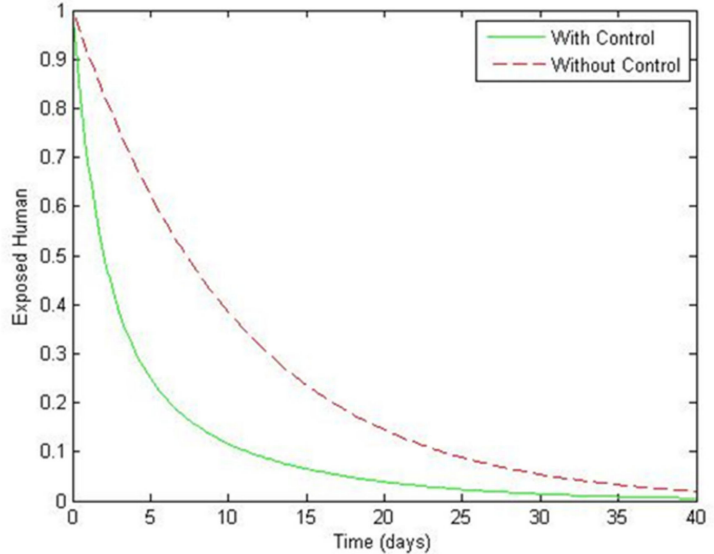

(b)

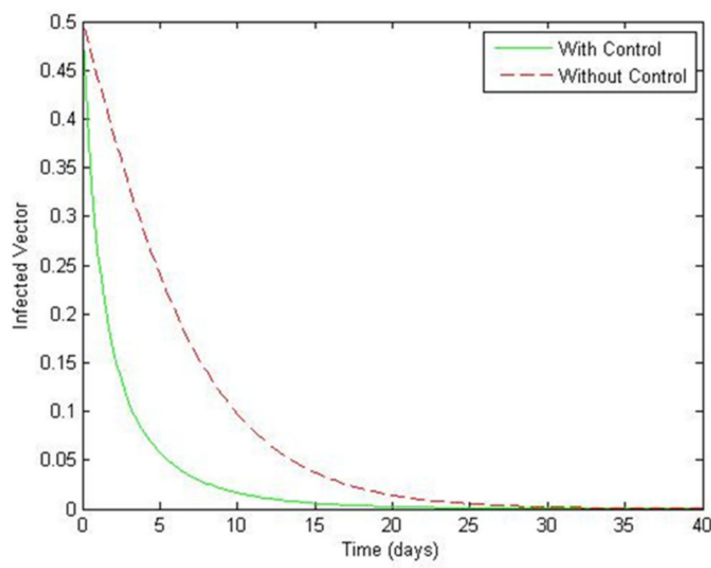

(d)

Figure 9. Comparison of behaviors with and without of system of Equations (33)-(36) of $S_{H}, E_{H}, I_{H}$, and $I_{V}$ when using $C_{1}=5, C_{2}=50$. (a) Susceptible human, (b) exposed human, (c) infected human, and (d) infected vector.

Figures 8-12 show the results of Equation (17) with and without control of $S_{H}, E_{H}, I_{H}$, and $I_{V}$. We have divided the weight simulations into five cases as follows: Case 1 represents the case of $C_{1}$ being much less than $C_{2}$; Case 2 represents the case of $C_{1}$ being less than $C_{2}$; Case 3 represents the case where both $C_{1}$ and $C_{2}$ are equal; Case 4 is where $C_{1}$ is greater than $C_{2}$ and Case 5 is where $C_{1}$ is much greater than $C_{2}$. The results of these cases are plotted in Figures 8-12, for Cases 1-5 respectively.

Figures 8-12 shows that after effective control such as for the human population is an effort to reduce the number of infected humans by giving vaccinations to humans and for the vector population is control by giving repellent to mosquitoes and destroying mosquito breeding sites. The number of the susceptible human $S_{H}$, exposed human $E_{H}$, infected human $I_{H}$, and infected vector $I_{V}$ is significantly reduced compared with that without control. The results of numerical simulation show that vaccination and the elimination of disease-carrying mosquitoes have a positive effect. The control, in particular, resulted in a significant reduction in the number of infected humans and vectors.

Figure 13a illustrates maintaining optimal control of vaccination rates in the human population $u_{1}(t)$ at initial value differences as follows: $40 \%, 60 \%$, and $80 \%$ in approximately the first 4 days control is maintained, then the control gradually decreases to zero. Figure $13 \mathrm{~b}$ shows the control values of the second control effort $u_{2}(t)$ at $40 \%, 60 \%$, and $80 \%$ initiation to achieve control. It is seen that the optimal level of control is maintained in the first 4 days, then gradually increases and reaches the plateau on Day 22, after which the control dosage is reduced to zero. 


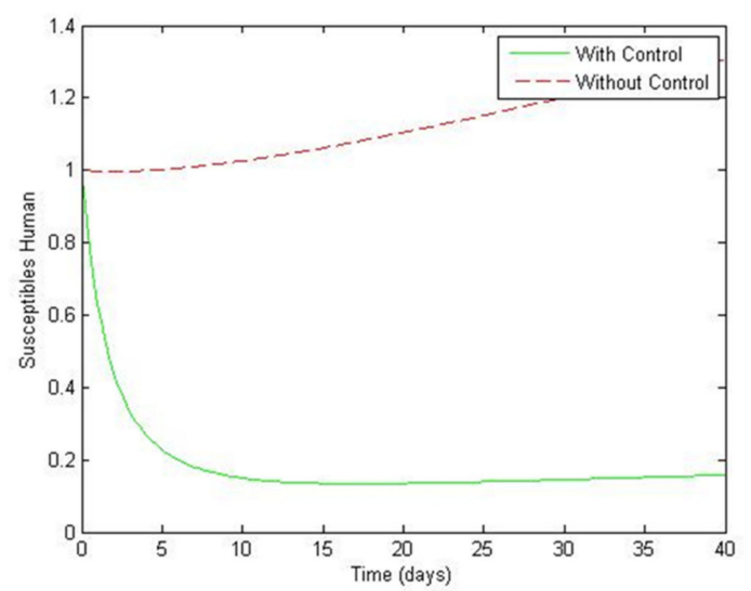

(a)

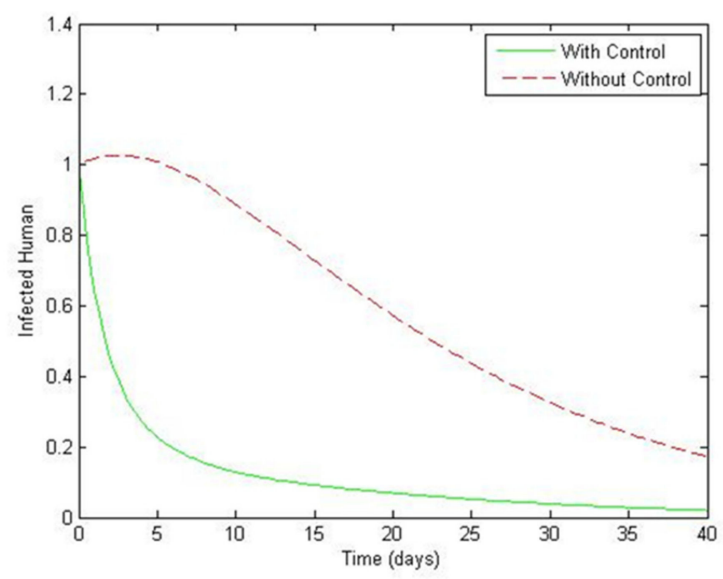

(c)

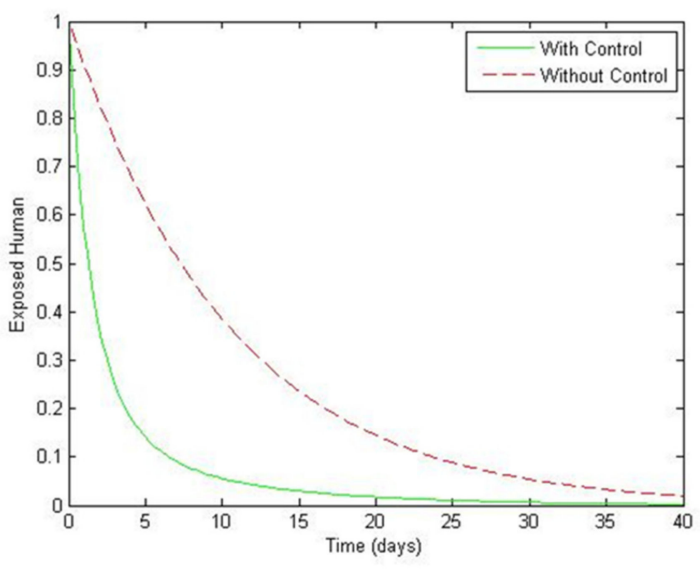

(b)

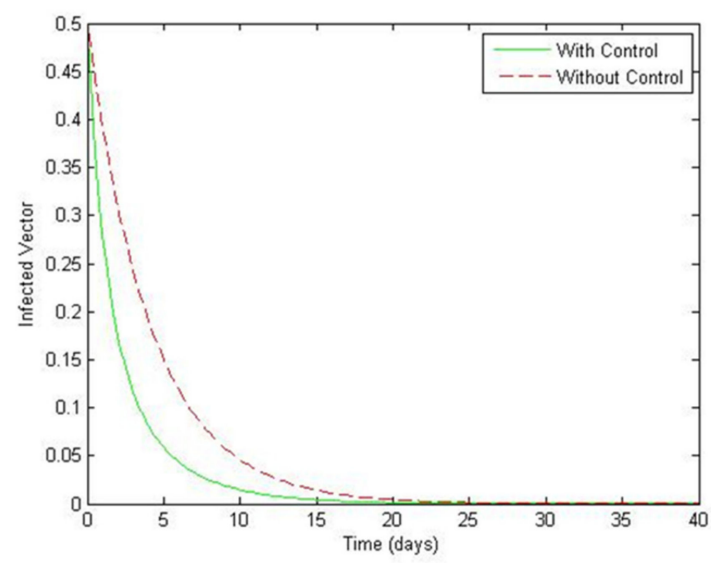

(d)

Figure 10. Comparison of behaviors with and without of system of Equations (33)-(36) of $S_{H}, E_{H}, I_{H}$, and $I_{V}$ when using $C_{1}=50, C_{2}=50$. (a) Susceptible human, (b) exposed human, (c) infected human, and (d) infected vector. 


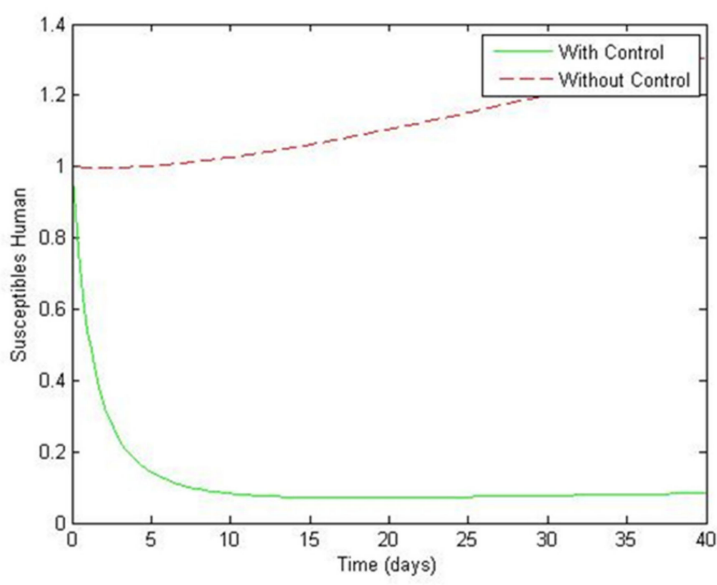

(a)

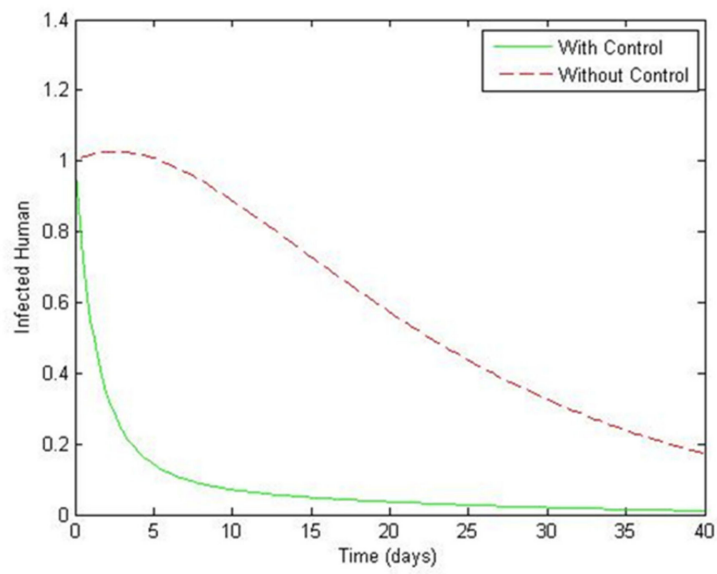

(c)

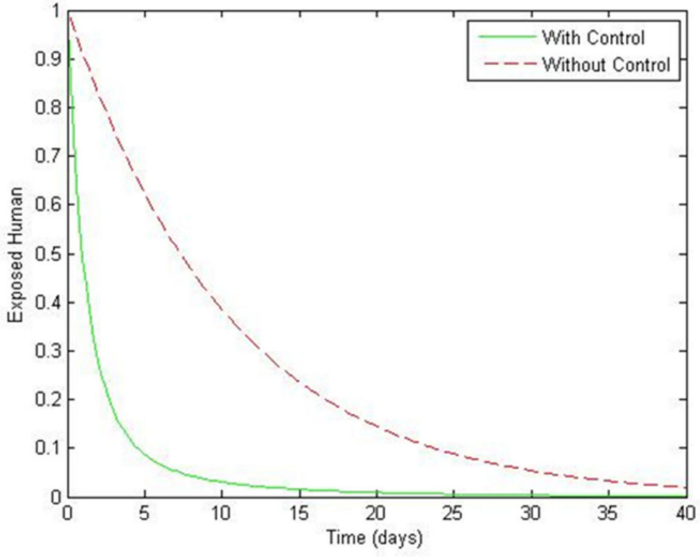

(b)

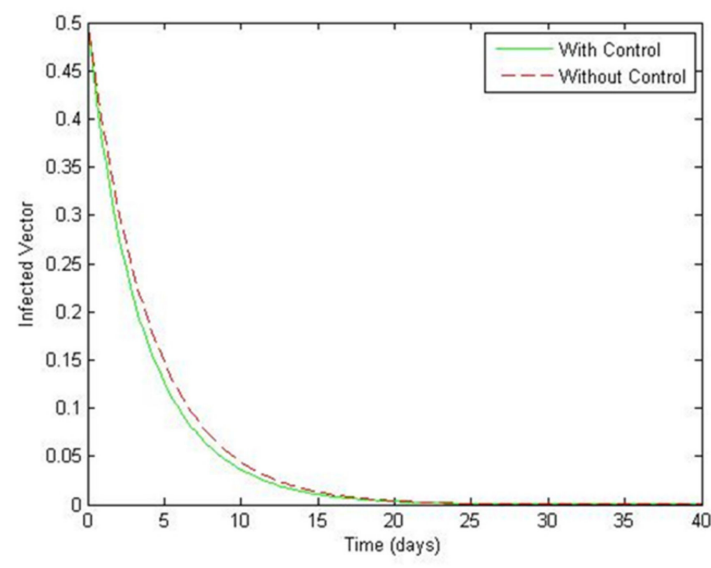

(d)

Figure 11. Comparison of behaviors with and without of system of Equations (33)-(36) of $S_{H}, E_{H}, I_{H}$, and $I_{V}$ when using $C_{1}=50, C_{2}=5$. (a) Susceptible human, (b) exposed human, (c) infected human, and (d) infected vector. 


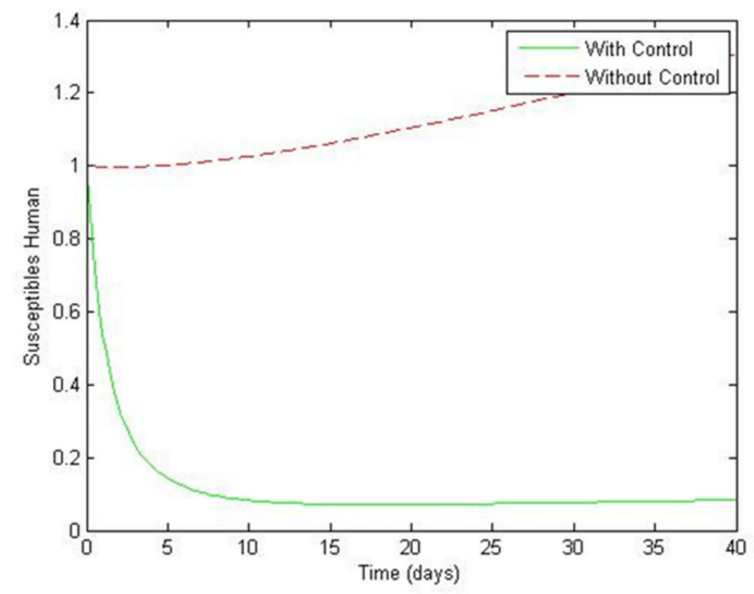

(a)

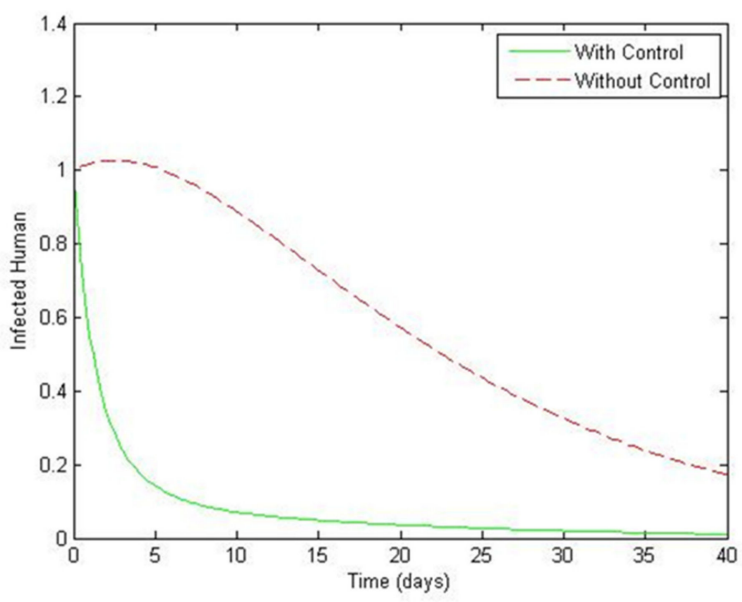

(c)

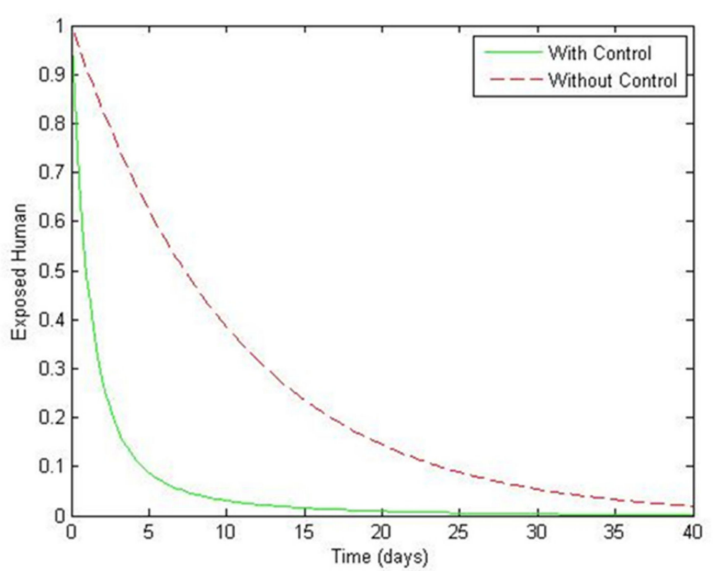

(b)

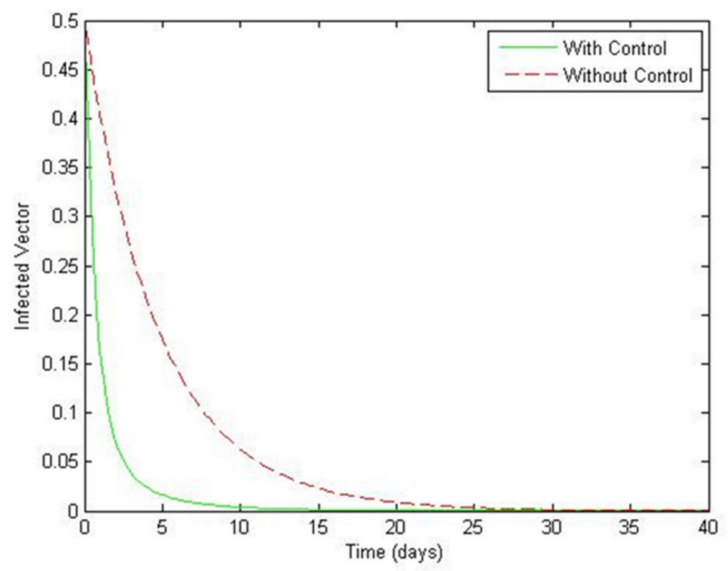

(d)

Figure 12. Comparison of behaviors with and without of system of Equations (33)-(36) of $S_{H}, E_{H}, I_{H}$, and $I_{V}$ when using $C_{1}=50, C_{2}=0.000005$. (a) Susceptible human, (b) exposed human, (c) infected human, and (d) infected vector.

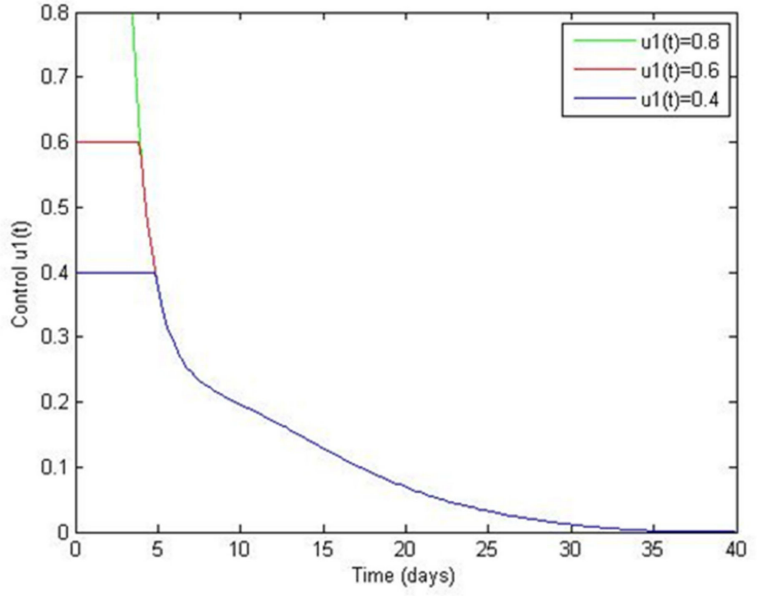

(a)

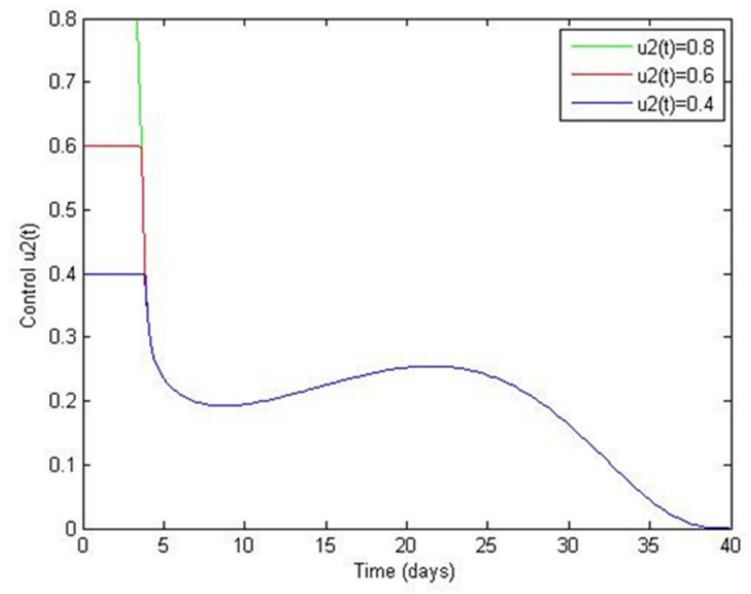

(b)

Figure 13. Control variables: (a) the vaccination rate $u_{1}(t)$ and $(\mathbf{b})$ the elimination of mosquitoes that carry diseases rate $u_{2}(t)$. Using $C_{1}=50, C_{2}=50$. 


\section{Conclusions}

In this article, we analyzed the impact of post-reinfection vaccination strategies for the second, third and fourth times, without regarding the serotype sequence during infection, for regions with high dengue outbreaks, in order to reduce the hospitalization rates and disease severity in the subsequent infections. This model is optimal for densely populated areas and abundant Aedes mosquitoes, which are carriers of dengue fever and there is a chance of re-infection with dengue fever multiple times. According to the 2020 report in Thailand, the region with the highest morbidity rate is the Northeastern region, with 127.53 per 100,000 inhabitants. The Routh-Hurwitz criterion for determining local asymptotically stability and the Lyapunov function for determining global asymptotically stability are used in the study. The equilibrium point that we found two states are disease-free converge to $E_{1}=(1,0,0,0)$ and endemic equilibrium point converge to $E_{2}=\left(S_{H}=0.00005, E_{H}=0.02689, I_{H}=0.01881, I_{V}=0.73048\right)$. The basic reproductive number is defined as $R_{0}$. The disease-free equilibrium point there exists locally asymptotically stable if $R_{0}$ is less than one and locally asymptotically stable when $R_{0}$ greater than one for the endemic equilibrium point is. Similarly, the disease-free and endemic equilibrium point exists globally asymptotically stable if and only if according to conditions of Theorems 3 and 4 . We simulated the numerical results solution of the compare two parameters with different values that affect the basic reproductive number value of this model as shown in Figures 6 and 7, where we can observe that the higher the dengue virus transmission rate from vector to human $\beta_{H}$ and the dengue virus transmission rate from human to vector $\beta_{V}$, the slower the convergence to a susceptible human equilibrium point. The exposed human, infected human, and infected vector all rapidly reach a point of equilibrium.

We developed the optimal control strategies using the elimination of mosquitoes that carry diseases rate and the vaccination rate to minimize the number of infected human population and infected vector population to cost controlling effort. The Pontryagin minimum principle (PMP) approach is used to solve the optimum control issue in this situation. We can see that effective measures such as vaccinations to humans and the annihilation of mosquito breeding sites represent powerful measures that effectively controlled the spread of the dengue virus. In comparison to those without control, the number of susceptible humans exposed, infected humans, and infected vectors are significantly reduced. The numerical simulation results show that the number of infections decreases over time. Figures 8-12 show that the control resulted in a significant reduction in the number of infected humans and infected vectors. Finally, the effective control and prevention of dengue fever rely on three components: improved vector control, improved case management, and effective vaccine development.

We suggest that future guidelines for this model should consider the serotypes of infection and provide a more complete age range for vaccination.

Author Contributions: Conceptualization, A.C., P.P. and N.W.; methodology, A.C.; software, N.W.; validation, A.C., P.P., I.-M.T. and N.W.; writing—original draft preparation, A.C.; writing-review and editing, A.C., P.P., I.-M.T. and N.W.; supervision, P.P., I.-M.T. and N.W.; project administration, P.P.; funding acquisition, P.P. All authors have read and agreed to the published version of the manuscript.

Funding: This research was funded by RA-TA graduate scholarship from the School of Science, King Mongkut's Institute of Technology Ladkrabang, Grant Number RA/TA-2562-D-035.

Institutional Review Board Statement: Not applicable.

Informed Consent Statement: Not applicable.

Data Availability Statement: Data are available from the corresponding author upon reasonable request.

Acknowledgments: This work is supported by the School of Science, King Mongkut's Institute of Technology Ladkrabang, Grant Number (2563-02-05-02).

Conflicts of Interest: The authors declare no conflict of interest. 


\section{References}

1. Remy, M.M. Dengue fever: Theories of immunopathogenesis and challenges for vaccination. Inflamm. Allergy Drug Targets 2014, 13, 262-274. [CrossRef]

2. Ministry of Public Health, Thailand. Dengue Fever. Available online: https://ddc.moph.go.th/disease_detail.php?d=44 (accessed on 20 June 2021).

3. World Health Organization. Dengue and Severe Dengue. Available online: https://www.who.int/news-room/fact-sheets/ detail/dengue-and-severe-dengue (accessed on 22 January 2021).

4. World Health Organization (WHO). Dengue: Guidelines for Diagnosis, Treatment, Prevention and Control: New Edition. Available online: https:/ /apps.who.int/iris/handle/10665/44188 (accessed on 15 September 2020).

5. Scott, T.W.; Amerasinghe, P.H.; Morrison, A.C.; Lorenz, L.H.; Clark, G.G.; Strickman, D.; Kittayapong, P.; Edman, J.D. Longitudinal studies of aedes aegypti (Diptera: Culicidae) in Thailand and Puerto Rico: Blood feeding frequency. J. Med. Entomol. 2000, 37, 89-101. [CrossRef]

6. Medlock, J.M.; Avenell, D.; Barrass, I.; Leach, S. Analysis of the potential for survival and seasonal activity of aedes albopictus (Diptera: Culicidae) in the United Kingdom. J. Vector Ecol. 2006, 31, 292-304. [CrossRef]

7. World Health Organization. Updated Questions and Answers Related to the Dengue Vaccine Dengvaxia ${ }^{\circledR}$ and its Use. Available online: https://www.who.int/immunization/diseases/dengue/QA_dengue_vaccine_22Dec2017.pdf (accessed on 15 August 2021).

8. World Health Organization. Comprehensive Guideline for Prevention and Control of Dengue and Dengue Haemorrhagic Fever. Revised and Expanded Edition. Available online: https:/ /apps.who.int/iris/handle/10665/204894 (accessed on 15 August 2021).

9. Kalayanarooj, S. Clinical Manifestations and Management of Dengue/DHF/DSS. Trop. Med. Health 2011, 39, S83-S87. [CrossRef] [PubMed]

10. Gubler, D.J. Dengue and Dengue Hemorrhagic Fever. Clin. Microbiol. Rev. 1998, 11, 480-496. [CrossRef] [PubMed]

11. Pongsumpun, P.; Tang, I. Transmission of Dengue Hemorrhagic Fever in an Age Structured Population. Math. Comput. Model. 2003, 37, 949-961. [CrossRef]

12. Sriprom, M.; Pongsumpun, P.; Yoksan, S.; Barbazan, P.; Gonzalez, J.P.; Tang, I.M. Dengue haemorrhagic fever in Thailand, 1998-2003: Primary or Secondary Infection. Dengue Bull. 2003, 27, 39-45.

13. Burke, D.S.; Scott, R.M.; Johnson, D.E.; Nisalak, A. A Prospective Study of Dengue Infections in Bangkok. Am. J. Trop. Med. Hyg. 1988, 38, 172-180. [CrossRef] [PubMed]

14. Villar, L.; Dayan, G.H.; Arredondo-Garcia, J.L.; Rivera, D.M.; Cunha, R.; Deseda, C.; Reynales, H.; Costa, M.S.; MoralesRamírez, J.O.; Carrasquilla, G.; et al. Efficacy of a Tetravalent Dengue Vaccine in Children in Latin America. N. Engl. J. Med. 2015, 372, 113-123. [CrossRef]

15. Sabchareon, A.; Wallace, D.; Sirivichayakul, C.; Limkittikul, K.; Chanthavanich, P.; Suvannadabba, S.; Jiwariyavej, V.; Dulyachai, W.; Pengsaa, K.; Wartel, T.A.; et al. Protective efficacy of the recombinant, live-attenuated, CYD tetravalent dengue vaccine in Thai schoolchildren: A randomised, controlled phase $2 b$ trial. Lancet 2012, 380, 1559-1567. [CrossRef]

16. World Health Organization. Fact Sheet: Questions and Answers on Dengue Vaccines: Phase III Study of CYD-TDV in Latin America. Available online: http://www.who.int/immunization/research/development/QA_Dengue_vaccine_LA_phIIIstudy_ final.pdf (accessed on 15 August 2021).

17. Hadinegoro, S.R.; Arredondo-Garcia, J.L.; Capeding, M.R.; Deseda, C.; Chotpitayasunondh, T.; Dietze, R.; Ismail, H.H.M.; Reynales, H.; Limkittikul, K.; Rivera-Medina, D.M.; et al. Efficacy and Long-Term Safety of a Dengue Vaccine in Regions of Endemic Disease. N. Engl. J. Med. 2015, 373, 1195-1206. [CrossRef]

18. Wilder-Smith, A. Dengue Vaccines: Dawning at last? Lancet 2014, 384, 1327-1329. [CrossRef]

19. World Health Organization. Fact Sheet: Global Strategy for Dengue Prevention and Control 2012-2020. Available online: http://www.who.int/immunization/sage/meetings/2013/april/5_Dengue_SAGE_Apr2013_Global_Strategy.pdf (accessed on 5 January 2021).

20. Esteva, L.; Vargas, C. Analysis of a dengue disease transmission model. Math. Biosci. 1998, 150, 131-151. [CrossRef]

21. Chanprasopchai, P.; Tang, I.M.; Pongsumpun, P. SIR Model for Dengue Disease with Effect of Dengue Vaccination. Comput. Math. Methods Med. 2018, 2018, 1-14. [CrossRef] [PubMed]

22. Phaijoo, G.R.; Gurung, D.B. Mathematical model of dengue fever with and without awareness in host population. Int. J. Adv. Eng. Res. Appl. 2015, 1, 239-245.

23. $\mathrm{Wu}, \mathrm{C}$; ; Wong, P.J.Y. Dengue transmission: Mathematical model with discrete time delays and estimation of the reproduction number. J. Biol. Dyn. 2019, 13, 1-25. [CrossRef]

24. Derouich, M.; Boutayeb, A. Dengue fever: Mathematical modelling and computer simulation. Appl. Math. Comput. 2006, 177, 528-544. [CrossRef]

25. Khan, M.A. Dengue infection modeling and its optimal control analysis in East Java, Indonesia. Heliyon 2021, 7, e06023. [CrossRef]

26. Pongsumpun, P.; Tang, I.M.; Wongvanich, N. Optimal control of the dengue dynamical transmission with vertical transmission. Adv. Differ. Equ. 2019, 176, 1-25. [CrossRef]

27. Chamnan, A.; Pongsumpun, P.; Tang, I.-M.; Wongvanich, N. Optimal Control of Dengue Transmission with Vaccination. Mathematics 2021, 9, 1833. [CrossRef] 
28. Xue, L.; Ren, X.; Magpantay, F.; Sun, W.; Zhu, H. Optimal Control of Mitigation Strategies for Dengue Virus Transmission. Bull. Math. Biol. 2021, 83, 1-28. [CrossRef] [PubMed]

29. Ndaïrou, F.; Torres, D. Pontryagin Maximum Principle for Distributed-Order Fractional Systems. Mathematics 2021, 9, 1883. [CrossRef]

30. Liu, G.; Chen, J.; Liang, Z.; Peng, Z.; Li, J. Dynamical Analysis and Optimal Control for a SEIR Model Based on Virus Mutation in WSNs. Mathematics 2021, 9, 929. [CrossRef]

31. Ndii, M.Z.; Mage, A.R.; Messakh, J.J.; Djahi, B.S. Optimal vaccination strategy for dengue transmission in Kupang city, Indonesia. Heliyon 2020, 6, e05345. [CrossRef] [PubMed]

32. Momoh, A.A.; Bala, Y.; Washachi, D.J.; Déthié, D. Mathematical analysis and optimal control interventions for sex structured syphilis model with three stages of infection and loss of immunity. Adv. Differ. Equ. 2021, 2021, 1-26. [CrossRef]

33. Ministry of Public Health Thailand. Dengue Fever. Available online: http://www.boe.moph.go.th/boedb/surdata/disease.php? dcontent $=$ old \&ds=66 (accessed on 5 January 2021).

34. Lamwong, J.; Wongvanich, N.; Tang, I.M.; Changpuek, T.; Pongsumpun, P. Global stability of the transmission of hand-foot-mouth disease according to the age structure of the population. Curr. Appl. Sci. Technol. 2021, 21, 351-369.

35. Prathumwan, D.; Trachoo, K.; Chaiya, I. Mathematical Modeling for Prediction Dynamics of the Coronavirus Disease 2019 (COVID-19) Pandemic, Quarantine Control Measures. Symmetry 2020, 12, 1404. [CrossRef]

36. Ajbar, A.; Alqahtani, R.; Boumaza, M. Dynamics of a COVID-19 Model with a Nonlinear Incidence Rate, Quarantine, Media Effects, and Number of Hospital Beds. Symmetry 2021, 13, 947. [CrossRef]

37. Edelstein-Keshet, L. Mathematical Models in Biology; SIAM: New York, NY, USA, 2005.

38. Basti, B.; Hammami, N.; Berrabah, I.; Nouioua, F.; Djemiat, R.; Benhamidouche, N. Stability Analysis and Existence of Solutions for a Modified SIRD Model of COVID-19 with Fractional Derivatives. Symmetry 2021, 13, 1431. [CrossRef]

39. La Salle, J.; Lefschetz, S. Stability by Liapunov's Direct Method with Applications; Academic Press: Cambridge, MA, USA, 1961.

40. Rouche, N.; Habets, P.; Laloy, M. Stability Theory by Liapunov's Direct Method; Springer: New York, NY, USA, 1977.

41. Sanusi, W.; Badwi, N.; Zaki, A.; Sidjara, S.; Sari, N.; Pratama, M.I.; Side, S. Analysis and Simulation of SIRS Model for Dengue Fever Transmission in South Sulawesi, Indonesia. J. Appl. Math. 2021, 2021, 1-8. [CrossRef]

42. Chien, F.; Shateyi, S. Volterra-Lyapunov Stability Analysis of the Solutions of Babesiosis Disease Model. Symmetry 2021, 13, 1272. [CrossRef]

43. Shang, Y. A lie algebra approach to susceptible-infected-susceptible epidemics. Electron. J. Differ. Equ. 2012, $2012,1-7$.

44. Shang, Y. Analytical Solution for an In-host Viral Infection Model with Time-inhomogeneous Rates. Acta Phys. Pol. B 2015, 46. [CrossRef]

45. Aguiar, M.; Stollenwerk, N.; Halstead, S.B. The Impact of the Newly Licensed Dengue Vaccine in Endemic Countries. PLoS Negl. Trop. Dis. 2016, 10, e0005179. [CrossRef]

46. Matheus, S.; Deparis, X.; Labeau, B.; Lelarge, J.; Morvan, J.; Dussart, P. Discrimination between primary and secondary dengue virus infection by an immunoglobuling avidity test using a single acute-phase serum sample. J. Clin. Microbiol. 2005, 46, 2793-2797. [CrossRef] [PubMed]

47. Shim, E. Optimal dengue vaccination strategies of seropositive individuals. Math. Biosci. Eng. 2019, 16, 1171-1189. [CrossRef] [PubMed]

48. Ndii, M.Z.; Allingham, D.; Hickson, R.; Glass, K. The effect of Wolbachia on dengue outbreaks when dengue is repeatedly introduced. Theor. Popul. Biol. 2016, 111, 9-15. [CrossRef]

49. Ndii, M.Z.; Allingham, D.; Hickson, R.I.; Glass, K. The effect of Wolbachia on dengue dynamics in the presence of two serotypes of dengue: Symmetric and asymmetric epidemiological characteristics. Epidemiol. Infect. 2016, 144, 2874-2882. [CrossRef]

50. Yang, H.M.; Macoris, M.L.G.; Galvani, K.C.; Andrighetti, M.T.M.; Wanderley, D.M.V. Assessing the effects of temperature on the population of Aedes aegypti, the vector of dengue. Epidemiol. Infect. 2009, 137, 1188-1202. [CrossRef]

51. Lukes, D.L. Differential Equations Electronics Resource: Classical to Controlled; Academic Press: London, UK, 1982.

52. Lenhart, S.; Workman, J.T. Optimal Control Applied to Biological Models; Chapman and Hall/CRC: Boca Raton, FL, USA, 2007. 